\title{
DOE/OR/00033-. T769
}

\author{
Determination of the Feasibility of Using Open Path FTIR to \\ Monitor Levels of 3-Methylfuran and 1-Octen-3-ol for the \\ Purpose of Detecting Microbial Contamination in Indoor \\ Environments
}

by

Brent Olive

\section{A RESEARCH PROJECT REPORT}

Submitted in partial fulfillment of the requirements for the degree of Master of Science in Public Health in the Department of Environmental Health Sciences in the School of Public Health, The University of Alabama at Birmingham.

\author{
BIRMINGHAM， ALABAMA
}

March, 1996

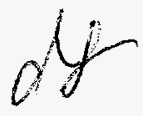




\section{DISCLAIMER}

This report was prepared as an account of work sponsored by an agency of the United States Government. Neither the United States Government nor any agency thereof, nor any of their employees, make any warranty, express or implied, or ascumes any legal liability or responsibility for the accuracy, completeness, or usefulness of any information, apparatus, product, or process disclosed, or represents that its use would not infringe privately owned rights. Reference herein to any specific commercial product, process, or service by trade name, trademark, manufacturer, or otherwise does not necessarily constitute or imply its endorsement, recommendation, or favoring by the United States Government or any agency thereof. The views and opinions of authors expressed herein do not necessar. ily state or rellect those of the United States Government or any agency thereof. 


\section{DISCLAIMER}

Portions of this document may be illegible electronic image products. Images are produced from the best available original document. 


\section{Abstract}

Studies have shown that the presence of microbial growth correlates with health complaints associated with sick building syndrome. Microbial growth may be found in damp places within a building, and may be dispersed to other areas if present in the HVAC system. Certain individuals may be especially sensitive to the presence of these microorganisms, and may experience adverse reactions at extremely low concentrations. Unfortunately, the source of the problem may not be discovered because many times the microbial growth is not visible. However, there are some volatile organic compounds that are given off by certain microorganisms which may be used to determine the presence of microbial contamination.

3-Methylfuran is an excellent indicator of growing fungi. It is produced by a majority of fungi, and can be used as an indicator of ongoing growth. 1-octen-3-ol is also produced by a number of fungi, and has been used in the past as an indicator of such. These two compounds and many other volatiles given off by microorganisms have been termed microbial volatile organic compounds (MVOCs). Many of these compounds are commonly found in indoor air, and thus, may be present even when there is not microbial contamination. The 
two MVOCs mentioned above are not commonly found in

buildings not contaminated with fungi; therefore, determination of these organic compounds can be used to confirm the presence of fungal contamination.

According to the current literature, the concentrations of 3-methylfuran and 1-octen-3-ol expected to be present in contaminated buildings are less than 0.007 and $1.9 \mathrm{ppb}$ respectively. To determine the feasibility of using open path FTIR to monitor for 3-methylfuran and 1-octen-3-ol, the LODs for the two compounds were determined by using the ETG software which is available with the instrument. Several spectra were collected in the absence of the target compounds over different path lengths and at different numbers of scans. The software was then used to calculate the lowest concentration that could be determined to be significantly different than the baseline noise of the spectra, or, the limit of detection. An LOD of 8 ppb was estimated for 3-methylfuran at a 100-m pathlength, which was the longest pathlength considered in this study due to the limitations that would normally be encountered when monitoring in indoor environments. The LOD for 1-octen-3-ol under the same circumstances was estimated to be $23 \mathrm{ppb}$. 
The LOD for 1-octen-3-ol is higher because its absorbtivity coefficient is less than that of 3-methylfuran.

At the present time, it does not seem feasible to use open path FTIR to monitor 3-methylfuran and 1-octen-3-ol for the purpose of confirming the presence of fungal growth. However, as technology improves, higher resolution instruments will be available, and if multiple reflection configurations were used, longer pathlengths could be achieved in indoor environments, both of which would result in lower LODs. 


\section{Acknowledgments}

I wish to thank the members of my research committee, Dr. Dillon, Chairman; Dr. Rose; Dr. Singh, and Don Gamiles for their assistance during the completion of this research project. I would like to especially thank Don for his continuing support in providing equipment and technical assistance in operating the FTIR Remote sensing unit.

I would also like to thank Mr. Joseph J. Vervier, Technical Director, Edgewood, Research Development and Engineering Center, and Dr. Clyde Frank, Deputy Assistant Secretary of Energy, for their sponsorship of my field work at the Center for NBC Environmental Technology. Their support was instrumental in the completion of this research effort.

The research was performed at the Center for NBC Environmental Technology under the appointment to the Industrial Hygiene Graduate Fellowship sponsored by the U.S. Department of Energy and administered by Oak Ridge Institute for Science and Education. 


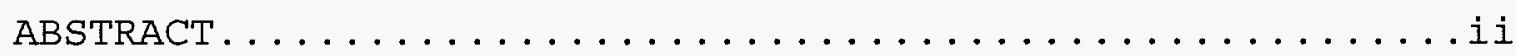

ACKNOWLEDGMENTS . . . . . . . . . . . . . . . . . .

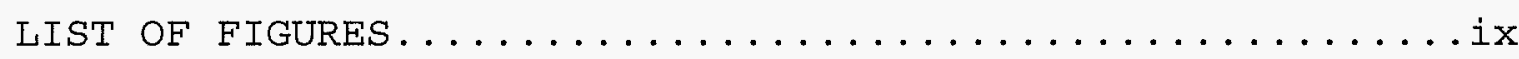

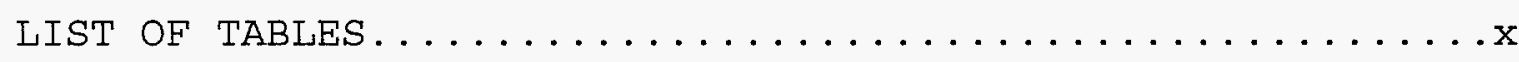

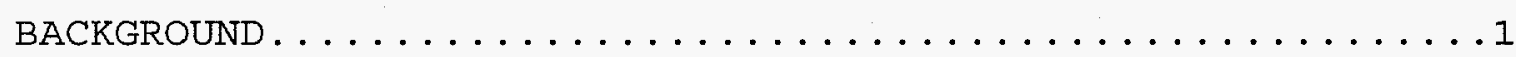

Infrared Absorption spectroscopy................

Theory of Infrared Absorption..............

Qualitative Applications of IR Spectroscopy.....4

Quantitative Applications of IR Spectroscopy....4

Open-Path Configurations...............

The Fourier Transform Infrared Remote Sensor........8

Advantages of Open-Path FTIR.............

Applications of OP-FTIR Spectroscopy..........10

Theoretical Description of FTIR Spectrometry.......11

The Michelson Interferometer.............11

Interference......................

Resolution.....................

The Detector.......................

The Infrared Source....................

The Data Output........................

Beer's Law....................... 
PAGE

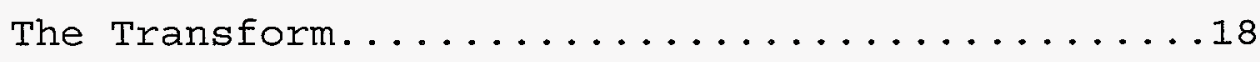

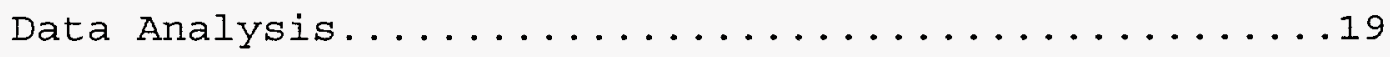

Generation of the Absorption Spectrum........19

Generation of the Reference Spectrum........20

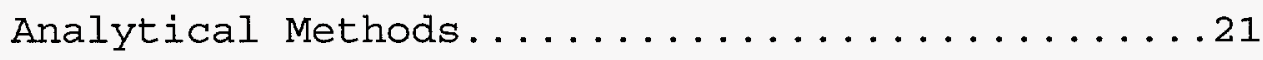

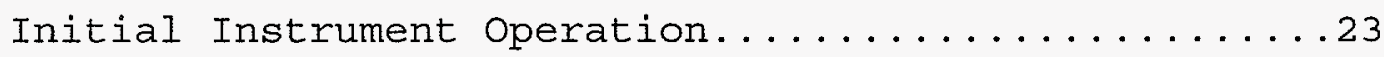

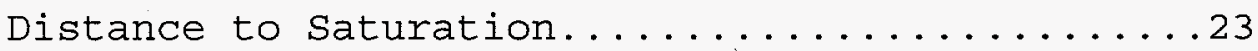

Return Intensity as a Function of Distance.....24

Determination of the Random Noise of the System.25

Return Intensity as a Function of Water Vapor...26

Background spectra...................... 27

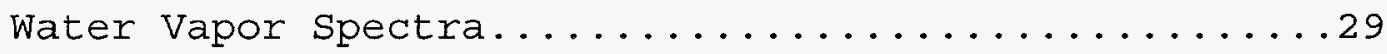

Resolution Considerations in Open Path Monitoring....32

Trading Rules in FTIR Spectrometry..........32

Effects of Resolution on Quantitative Analysis..35

Microbial Volatile Organic Compounds (MVOCs) ........38

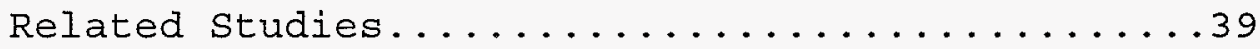

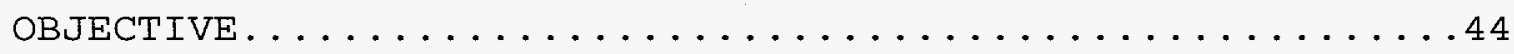

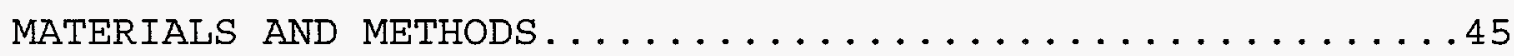

Discription of the Instrument.............

Development of Reference Spectra...............

Field Tests........................48 


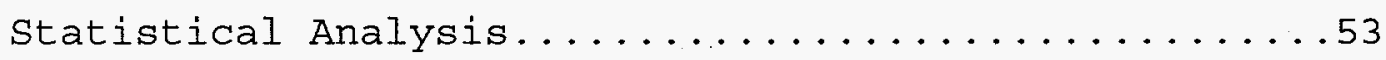

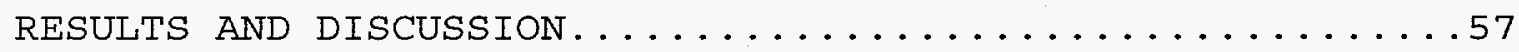

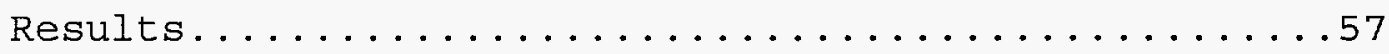

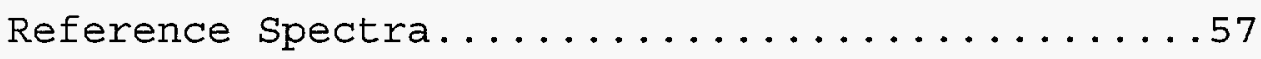

Limits of Detection...............62

Discussion..........................

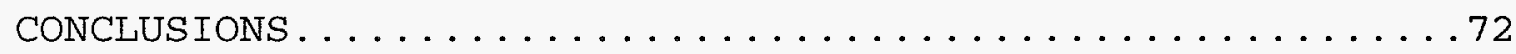

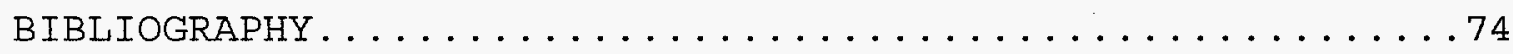

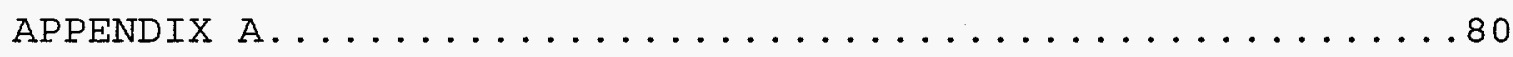

APPENDIX 


\section{LIST OF FIGURES}

FIGURE

PAGE

1. Monostatic configuration $1 \ldots \ldots \ldots \ldots \ldots$

2. Monostatic configuration $2 \ldots \ldots \ldots \ldots \ldots$

3. Simplest form of a Michelson interferometer....12

4. Center burst of an interferogram...........14

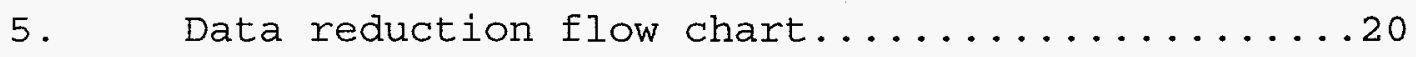

6. Reference spectrum for 3 -methylfuran........58

7. Reference spectrum for 1 -octen-3-ol.........59

8. Region of interest (3-methylfuran)........60

9. Region of interest (1-octen-3-ol) .........61

10. Affects of scan time... on LoDs (3-methylfuran).63

11. Affects of scan time... on LoDs (1-octen-3-ol)..63 


\section{LIST OF TABLES}

TABLE

I. Summary of LoDs for Original Data.........62

II. Summary of LODs for $34 \%$ RH Data..........64

III. Summary of LODs for $77 \%$ RH Data..........64 


\section{BACKGROUND}

\section{Infrared Absorption Spectroscopy}

Infrared spectroscopy has been used in the past primarily as a qualitative analytical tool, but is increasingly being used for quantitative analysis as well in a number of applications. Modern instruments are portable and are becoming useful industrial hygiene tools. The Miniature Infrared Analyzer (MIRAN), a dispersive infrared spectrophotometer, has been used in many industrial applications over the past few years. Infrared instruments which apply a Fourier Transform have also been frequently used. In one study, the MIRAN and a portable FTIR system were used in the determination of concentrations of trichloroethylene in an industrial setting, while in the presence of Freon-113 (1). Techniques involving FTIR have also been used in environmental monitoring of smog in Los Angeles (2), and in ambient air studies involving the direct measurement of solvents in breath in order to compare the concentrations with that of ambient air (3). There are many 
other applications of infrared absorption spectroscopy, all of which are based on the same fundamental principles of infrared absorption.

Theory of Infrared Absorption

The infrared spectrum is divided into near-, mid-, and far-infrared regions (4). The near region corresponds to wavenumbers ranging from $12,800-4000 \mathrm{~cm}^{-1}(0.78-2.5 \mathrm{um})$, the middle region ranges from 4000-200 $\mathrm{cm}^{-1}(2.5-50 \mathrm{um})$, and the far region is 200-10 $\mathrm{cm}^{-1}(50-1000 \mathrm{um})$. The middle infrared region is where most of the fundamental bands of molecules fall, and is generally the most useful part of the spectrum for atmospheric analysis (5). Compounds whose mid-infrared spectra have numerous maxima and minima can be distinguished from the absorption patterns of other compounds (4). Only optical isomers would absorb in exactly the same way and have identical absorption spectra.

In order for absorption of infrared radiation to occur, a molecule must undergo a net change in dipole moment which is caused by its vibrational or rotational motion (4). The dipole moment is determined by the magnitude of the difference in charge and the distance between the two centers of charge. As a molecule vibrates, a fluctuation in dipole moment occurs which establishes a field that can 
interact with the electrical field associated with the radiation. If the frequency of the radiation matches the vibrational frequency of the molecule, a change in the amplitude of the molecular vibration takes place due to a net transfer of energy. Absorption of radiation occurs as a consequence of these events. In a similar way, the rotation of asymmetric molecules around their centers results in a dipole fluctuation that can interact with radiation. Homonuclear species such as the diatomic molecules $\mathrm{O}_{2}, \mathrm{~N}_{2}$, and $\mathrm{Cl}_{2}$, experience no net change in dipole moment during rotation or vibration, and thus, cannot absorb in the infrared region. All other molecules that are not of this type absorb infrared radiation.

There are two types of vibrations that cause change in dipole moment: stretching and bending (4). Stretching vibrations cause a continuous change in the interatomic distance along the axis of the bond between two atoms. Bending vibrations involve the changing of the angle between two bonds. There are four different types of bending vibrations: scissoring, rocking, wagging, and twisting. All of these bending vibrations may possibly occur in a molecule which contains more than two atoms. 
Qualitative Applications of Infrared Spectroscopy

Identification of compounds from spectra can generally

be described by a two-step process (4). The first step involves determining what functional groups are most likely present by examining the frequency regions where they are known to absorb. The second step is to compare the spectrum of the sample with spectra of pure compounds that contain all of the functional groups found to be present in step one. Traditionally, a portion of the mid-infrared, i.e., the region from $1200 \mathrm{~cm}^{-1}$ to $600 \mathrm{~cm}^{-1}$, is known as the fingerprint region because small differences in the structure of a molecule result in significant changes in the appearance and distribution of absorption peaks in this spectral range. A close match between two spectra in the fingerprint region is almost certain evidence that the two compounds producing the spectra are identical. Computerized techniques of comparing spectra have been developed and will be discussed in subsequent sections of this text.

Quantitative Applications of Infrared Spectroscopy

Quantitative analysis by infrared absorption spectroscopy is based on the assumption that absorbance is directly proportional to the concentration of the analyte present (4). There are several different types of infrared 
instruments that can be used for quantitative analysis which operate on this principle. One such instrument is a dispersive infrared spectrophotometer. Dispersive instruments are generally double-beam instruments which use reflection gratings for dispersing radiation. Another type of instrument is a nondispersive photometer which often uses simple filters or may not employ any wavelength restricting device at all. Even though these instruments were commonly used in the past, another type of infrared instrument known as a Fourier transform infrared spectrometer is receiving increased usage.

Fourier transform infrared spectrometers are multiplex instruments which means that all elements of the signal are observed simultaneously (4). In order to determine the magnitude of each element of the signal, it is necessary to modulate the signal in a way that permits subsequent decoding of the signal. In these particular instruments, the Fourier transform is used for the data processing as the name implies. Traditionally, these instruments have used a closed-cell system in which the sample is introduced. The infrared beam then passes through the cell to allow absorption to take place. The beam may pass through the cell only once, or may be reflected multiple times if a 
longer path length is desired. The closed-cell system is still the most commonly used configuration today, but another technique known as open-path FTIR spectroscopy has become an alternative configuration.

Open-Path Configurations

There are two primary configurations which are used for transmitting the infrared beam in OP-FTIR spectroscopy (6). One is the monostatic system which has both the IR source and the detector at the same end of the path. The other configuration is the bistatic system which has the detector at one end of the path and the source at the other end. In the bistatic system, the optical path length is equal to the physical path length between the source and the detector. In the monostatic system, the optical path length is equal to twice the physical path length between the instrument and the retroreflector.

There are two different configurations commonly used with the monostatic system (6). The two configurations differ in the way that the infrared beam is returned along the optical path. The first configuration, shown in Figure 1, translates the infrared beam slightly so that it does not fold back on itself. The receiving telescope is slightly 
removed from the transmitter with the detector at the primary focus.

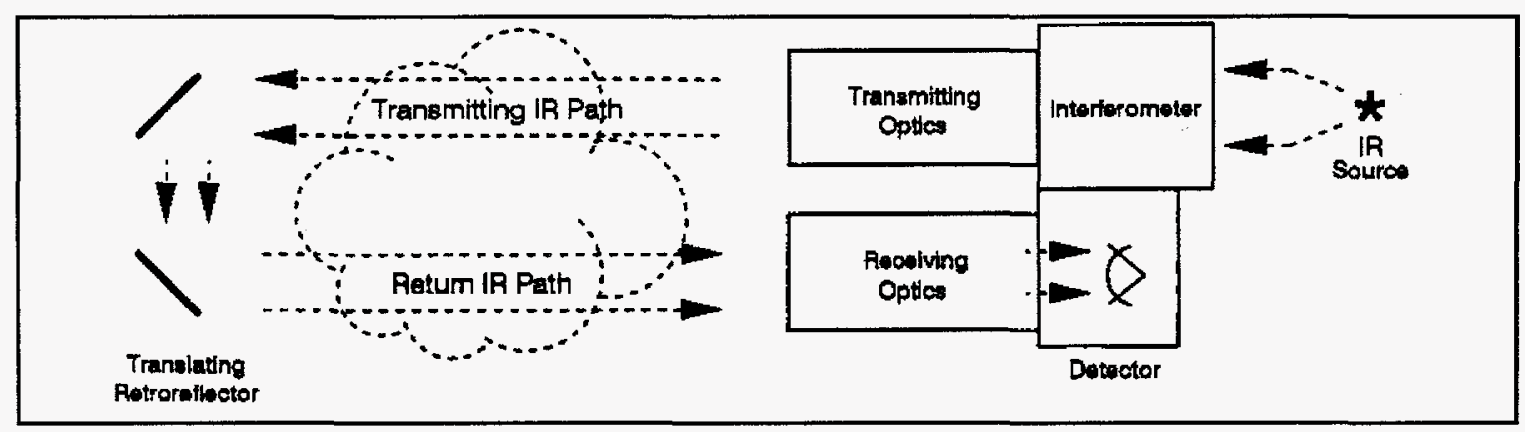

Figure 1. Monostatic configuration 1 (taken from
Reference 6 with permission) The second monostatic configuration uses the same telescope for the transmitting and the receiving optics (6). This configuration is illustrated in Figure 2. The beam strikes a retroreflector, and is returned to the instrument along the same path. The retroreflector is an arrangement of three mutually perpendicular mirrors, and is commonly called a corner cube. This design makes alignment of the beam much simpler than it would be with a flat mirror because the individual cubes efficiently reflect the infrared beam even when it is not perfectly alligned with the retroreflector. 


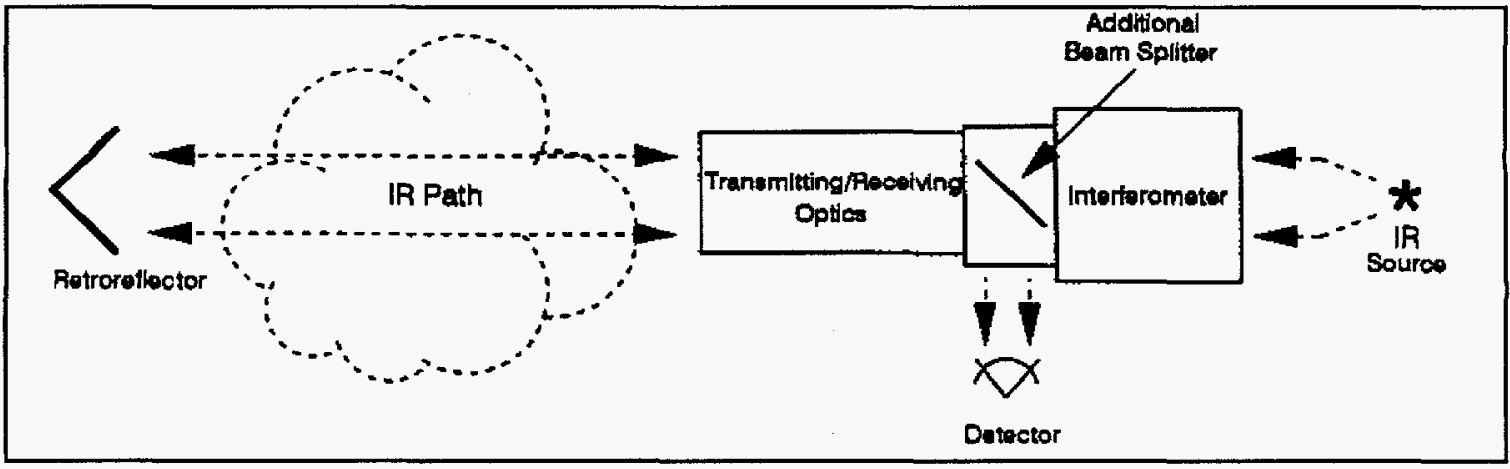

Figure 2. Monostatic configuration 2 (taken from Reference 6 with permission)

One drawback to using the same optics for transmitting and receiving is the fact that a beam splitter must laced in the optical path (6). The infrared beam passes through the beam splitter when it is transmitted and when it is received. The most effective beam splitter transmits $50 \%$ of the light and rejects the other $50 \%$, thus, after the two passes through the beam splitter, the transmission will only be $25 \%$ of the original beam which decreases the signal-tonoise ratio. However, when the path lengths used are relatively short, such as with indoor use, the problem of detector saturation far exceeds that of transmission loss, so in certain circumstances this loss in transmission will not affect the results.

\section{The Fourier Transform Infrared Remote Sensor}

The use of open-path infrared instruments for monitoring atmospheric pollutants was originally discussed 
by Philip L. Hanst in 1970 (5). One of the original

instruments was a dispersive instrument called the ROSE (Remote Optical Sensing of Emissions) system which was completed in 1972 (7). In 1977, the existing device was converted to an FTIR system which greatly increased the resolution, and thus, the sensitivity of the instrument. However, the instruments used in the 1970's were large and had to be installed in vans in order to make them mobile (8). Although largely successful in applications, the usage of open path FTIR in environmental monitoring was minimal until the late $1980^{\prime} \mathrm{s}$ (6). Today, progress is continuing, and there is still a great deal of work being done to improve open-path monitoring techniques.

Advantages of Open-Path FTIR

The two most widely used methods of air monitoring today are personal and general area sampling. Personal sampling has an advantage over general area sampling in that the monitor moves with the person and therefore, gives a better measure of breathing zone exposure (9). However, personal sampling also has drawbacks such as the usual requirement for laboratory analysis which may take the lab days, or even weeks to perform. General area monitoring can be done with direct reading instruments which measure 
exposures in real-time, but temporal resolution is often gained at the expense of spatial resolution. Also, many direct reading instruments are not selective, and therefore, their use in industry may be limited to certain applications such as monitoring total hydrocarbon levels.

OP-FTIR spectroscopy can give the desired temporal resolution of direct reading instruments, while providing the selectivity necessary to monitor multiple compounds simultaneously (9). Also, integrated path concentrations may be a better indicator of spatial dispersion as opposed to point sampling with a direct reading instrument. When combining the sensitive and specific chemical detection capabilities of optical remote sensing with the ability to integrate over space, "topographical" maps of chemical concentrations in a room can be produced (10). Thus, if one were interested in monitoring departures from normal conditions in a room, the OP-FTIR has the ability to provide information that neither personal nor area sampling can (9). Applications of OP-FTIR Spectroscopy

There are many potential uses of OP-FTIR monitoring. One of the current interests is to use the technique to measure fugitive emissions such as spills and leaks, in order to alert people in the vicinity of hazardous waste 
sites or other areas producing such emissions. Also, there is an interest in using the technique for measuring stack emission rates. One such study involving measurement of stack emissions was conducted with the ROSE system in the early $1980^{\prime}$ 's which showed that OP-FTIR spectroscopy can be a valuable tool for such analyses (11).

Theoretical Description of FTIR Spectrometry The Michelson Interferometer

The Michelson interferometer is the primary optical component in a FTIR instrument (6). The trace of the output of the interferometer is called the interferogram. The interferogram contains all of the information about the spectrum, but is not in a form that is readily recognizable to most spectroscopists. The interferometer consists of a beam splitter and two mirrors, one stationary, and one which is moveable. The mobile mirror moves along the optic axis while staying perpendicular to the fixed mirror at all times. The requirement for this perpendicularity limits the amount of movement of the mirror. The incident beam on the splitter is collimated to give better resolution. Figure 3 is a schematic of a Michelson interferometer. 


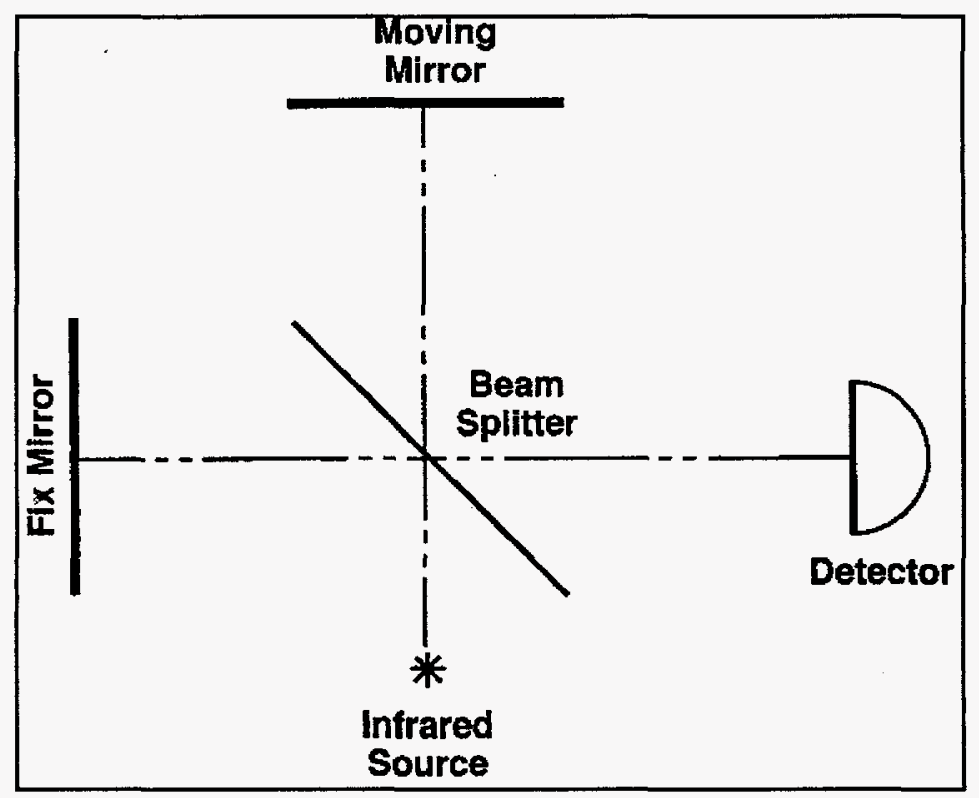

Figure 3. Simplest form of a Michelson interferometer (taken from Reference 6 with permission)

Interference

The underlying physical phenomenon that allows a Fourier transform instrument to obtain spectrometric data is the interference of light waves (6). The amplitude of interfering waves is calculated by adding the amplitudes of the individual beams according to the principle of superposition. Interference phenomena are linear in amplitude because the principle of linear superposition follows directly from a set of linear differential equations known as Maxwell's equations. 
If a source emits a plane wave whose amplitude is

divided into two separate beams, the individual beams can be recombined at a point $P$ in space (6). By using the relationship $2 \cos x=e^{i x}+e^{-i x}$, the intensity at point $\mathrm{P}$ can be given by the following equation.

$$
I(P)=2 I_{0}\left\{1+\cos \left[2 \pi n\left(T_{2}-T_{1}\right) / \lambda\right]\right\}
$$

Here $I_{0}$ is the intensity of either beam. As the difference of the path length, $T_{2}-T_{1}$, changes, the intensity at point $P$ can vary from 0 up to $4 I_{0}$. This does not violate the law of conservation of energy because there is no requirement that the intensity at every point be $2 I_{0}$, but rather that the interference term averaged over space must equal zero. Ideally, a plane wave will be divided evenly in intensity when sent through the beam splitter of the Michelson interferometer (6). The detector records the integral of the intensities over wavelength at any given position of the moving mirror. This recording is called the interferogram. When the difference in path is equal to zero, the cosine term will be one, and the intensity will be $4 I_{0}$ for all wavelengths. At this point, the output of the detector is large compared to any other detector position, which produces a noticeable change in the interferogram called a 
center burst. Figure 4 shows an example of the center burst of an interferogram.

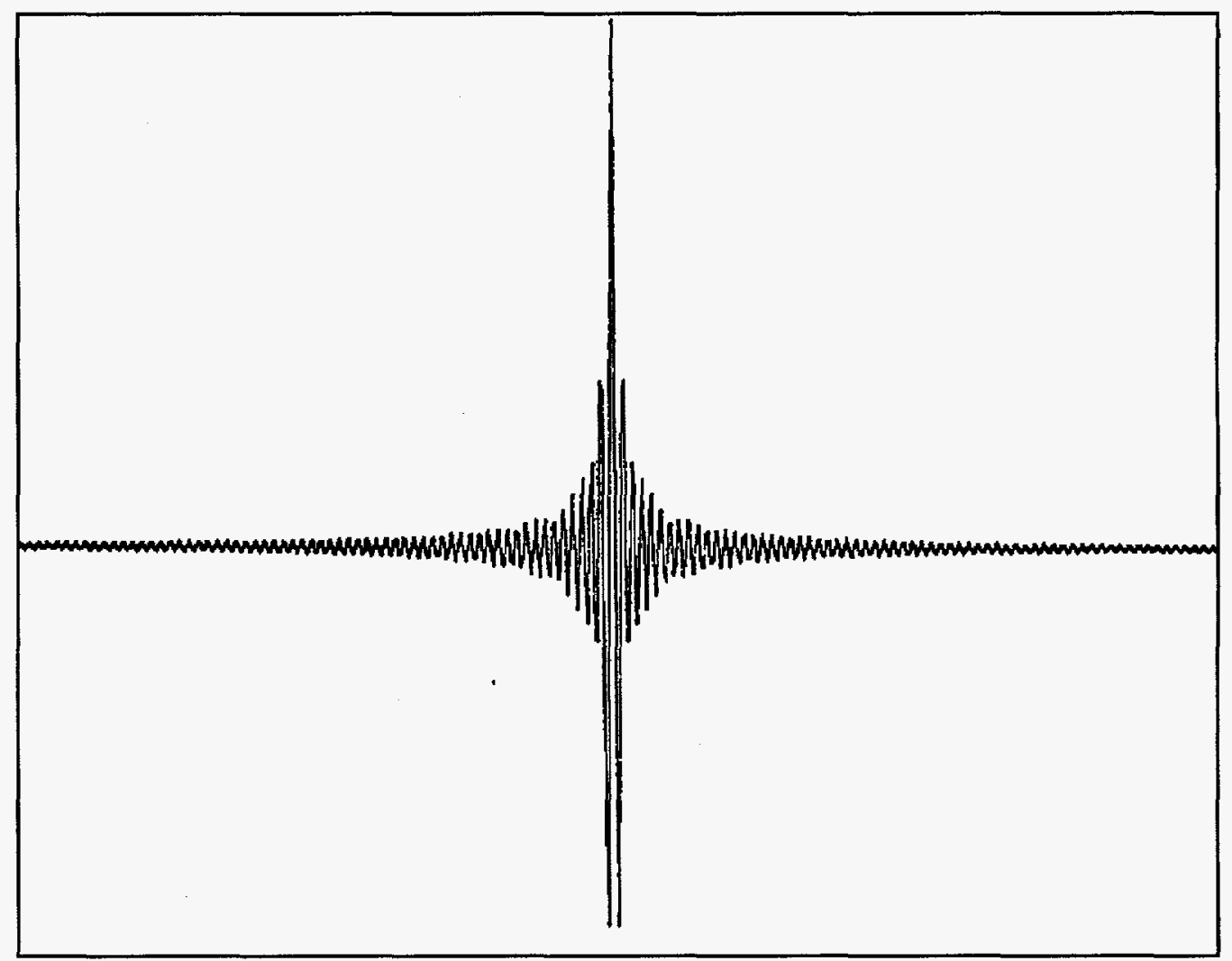

Figure 4. Center burst of an interferogram (taken from Reference 8 with permission)

As the mirror moves, the path difference $\left(T_{2}-T_{1}\right)$ causes a modulation of the intensity at each wavelength, which is shown by Equation 1 (6). This modulation is most advantageous if the infrared beam is sent through the interferometer before it is sent along the open path. By using this configuration, background radiation entering the system from the surroundings will not be modulated by the 
interferometer. However, the unmodulated background radiation may still reach the detector, and if the source is bright enough, it could cause the detector to become saturated.

\section{Resolution}

The resolution of the FTIR spectrometer can be defined as the inverse of the length the mobile mirror travels beyond the zero path difference, which is expressed in reciprocal centimeters or wave numbers (12). The resolution determines how close two absorption features can be, and still be separated enough that the instrument will be able to distinguish one from another (6). It can be shown by Equation 1 that all wavelengths have a maximum and are in phase with one another at zero path difference. If there are two absorbing features centered at wavelengths $\lambda_{1}$ and $\lambda_{2}$, they will be resolved if the mirror moves at least to the point where these two wavelengths are again in phase. The Detector

Most FTIR instruments used for monitoring atmospheric pollutant gases are equipped with a semiconductor device made of mercury, cadmium, and telluride which is commonly known as a MCT detector (6). The MCT detector converts a beam of photons to an electrical signal that can be 
measured. Noise equivalent power (NEP), the sensitivity of the detector, and the spectral range are the most important parameters.

It has been shown that detectors cooled to liquid nitrogen temperatures have a much greater sensitivity as compared to those that operate at room temperature (13). One current technique for cooling the detector is to place it in a Dewar that uses liquid nitrogen as a refrigerant (6). The Dewar must be filled periodically in order to maintain the proper level of liquid nitrogen. Another technique is to use a cryogenic cooler such as a stirling engine. This cooling device has a limited lifespan and may add noise to spectra, but if unattended operation is necessary, this method may be the best choice.

\section{The Infrared source}

Infrared sources used in FTIR instruments are heated elements which are exposed to the open environment. One of the most detrimental characteristics of the infrared sources that are available today is the fact that they are large in comparison to the focal length of the collimating optics (6). This means that the beam can never be collimated better than the angle at the retroreflector. As a result, retroreflectors become saturated and cannot reflect all of 
the incident beam and much of the initial energy is lost.

Smaller sources that are capable of producing the same heat intensities would help solve this problem, and possibly allow for measurement over longer path lengths.

\section{The Data Output}

Beer's Law

All. IR quantitative analyses are based on what is known as Beer's law, which states that, for a constant path length, the intensity of the incident beam energy traversing an absorbing medium diminishes exponentially with

concentration (6). Beer's law is mathematically defined by the following equation.

$$
I(v)=I_{0}(v) e^{-\alpha(v) C L}
$$

$I_{0}(v)$ is the intensity of he incident beam, $\alpha$ is the optical absorption coefficient of the absorbing material as a function of wave number $v, C$ is the concentration of the target gas, and $L$ is the path length. Regardless of the units chosen, the product of $\alpha C L$ should be a unitless quantity.

Beer's law is one of the basic principles of FTIR analysis. If $\alpha$ is known (which sometimes is the case), this principle can be directly applied to FTIR applications (6). One implication of Beer's law that is used directly is the 
concept of reciprocity, which states that if the

concentration decreases by a factor of two, and the path

length increases by a factor of two, the outcome will be the same. Reciprocity does not always hold true, and it is generally accepted that if the quantity $\alpha C L$ exceeds 0.1 , the concept is no longer valid.

\section{The Transform}

A sum of sine and cosine terms is known as a Fourier series and is used to describe such items as vibrating strings or other periodically varying quantities (6). The sine and cosine terms have frequencies at which an item or quantity can vibrate, which are known as fundamental frequencies. A harmonic analysis of the original vibratory motion is a linear combination of these fundamental frequencies which reveals that there are related pairs of variables such as time and frequency, or position and momentum. Functions can also be analyzed by applying a general Fourier transform. The Fourier series can be used to describe a periodic function as an infinite sum of sine and cosine terms, as well as for the analysis of nonperiodic functions as an integral over a continuous range of frequencies. Equation 4 shows the relationship between time and frequency. 


$$
\mathscr{F}(\omega)=1 /(2 \pi)^{0.5} \int G(t) e^{-i \omega t d t}
$$

The Fourier transform $\mathscr{F}(\omega)$, is a function of frequency and is related to $G(t)$, which is a function of time. The function $G(t)$ is the interferogram produced by the system, and $\mathscr{F}(\omega)$ is referred to as the single beam spectrum. In this case, the $t$ in the term $G(t)$ is related to the position of the moving mirror from the center burst position.

\section{Data Analysis}

\section{Generation of the Absorption Spectrum}

Analysis of data generally starts with the recording of the interferogram. The interferogram can be used directly to determine the concentration of the desired analyte, but more often the interferogram is converted into what is called a "single-beam spectrum" by the Fourier transform (6). A single-beam spectrum is produced and recorded for each sampling period and is sometimes referred to as a field or analytical spectrum. A background spectrum is produced and then a transmission spectrum is obtained by dividing the analytical spectrum by this background. If the background spectrum is taken appropriately, the transmission spectrum is analogous to a double beam spectrum taken with a conventional double beam IR spectrophotometer. Taking the negative logarithm of the transmission spectrum produces the 
absorption spectrum which is used for all of the further data analysis. This process is illustrated in Figure 5.

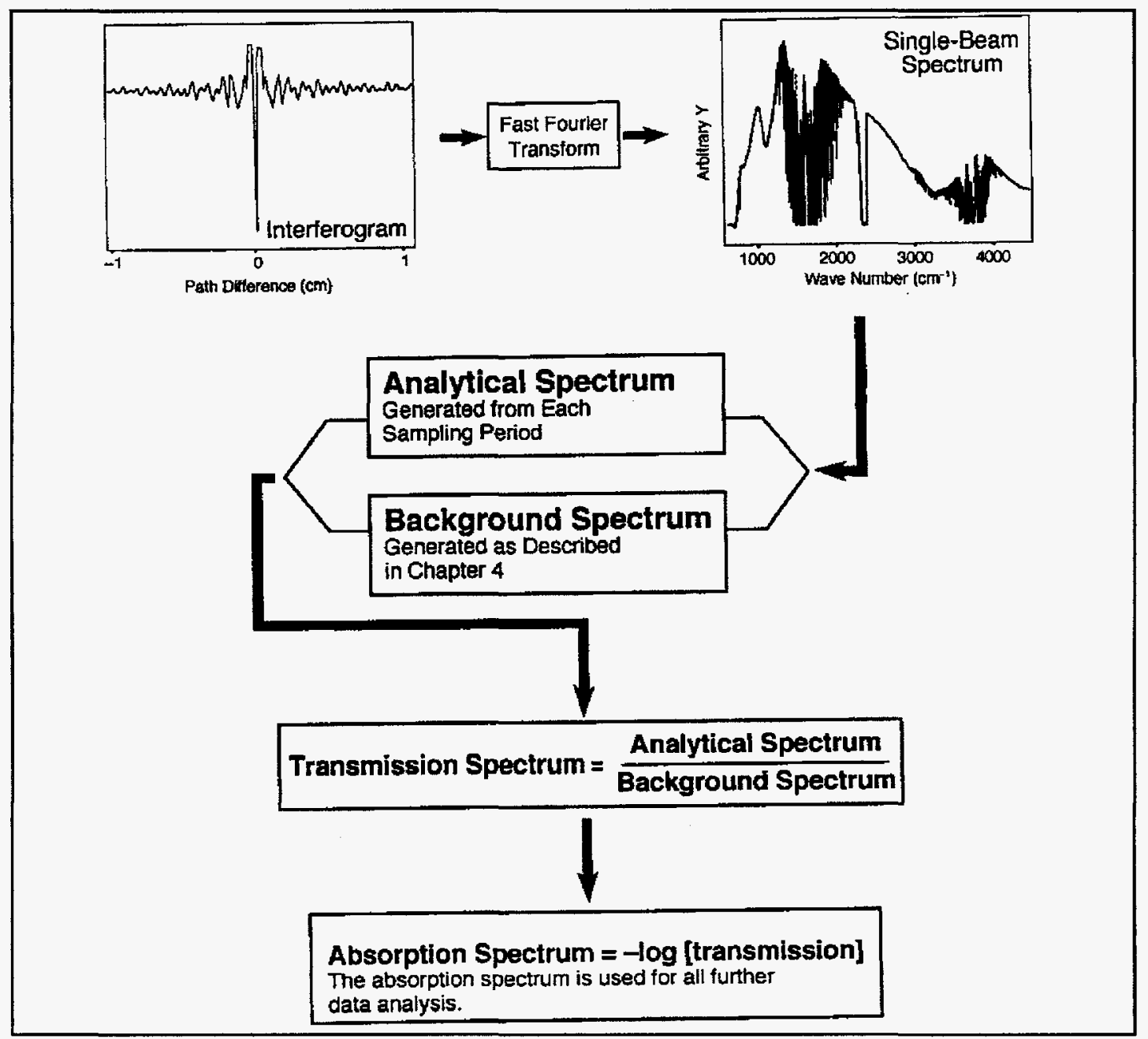

Figure 5. Data reduction flow chart (taken from Reference 6 with permission)

\section{Generation of the Reference Spectrum}

A reference spectrum can be generated in a relatively short cell (about one meter), or a multipass cell can be used which has a significantly longer path length (ten 
meters or more) (6). One of the more common methods is to mix the pure gas with an inert gas so that the internal pressure of the cell is approximately equal to atmospheric pressure (12). In the past, an evacuated cell has also been used to generate reference spectra, but this method often produces a baseline shift, probably due to a slight distortion of the walls of the cell when internal pressures are extremely low. The production of reference spectra is not a simple task and great attention must be paid to the details of the experimental setup (13). It should be noted that there are no prescribed procedures for this process because most users of the FTIR instrument do not prepare their own reference spectra (6).

\section{Analytical Methods}

Once the reference absorption spectrum is obtained, the appropriate wave number region to be used for analysis must be selected based on two criteria: the gas should have a high absorption coefficient in the selected region, and the region should be free of absorption bands from interfering species (6). Usually interfering species are present, and they must be accounted for in the analysis technique.

One method of determining the concentration of the target gas is the comparison technique (6). The comparison 
technique involves measuring the absorbance at a particular wave length and comparing it with the absorbance of the reference spectrum at the same wave number. If reciprocity holds, the concentration is determined by the following equation.

$$
A_{\text {ref }} / A_{\text {unk }}=C_{\text {ref }} L_{\text {ref }} / C_{\text {unk }} L_{u n k}
$$

Equation 5 can then be solved for the unknown concentration to give Equation 6 .

$$
C_{\text {unk }}=C_{\text {ref }} L_{\text {ref }} A_{\text {unk }} / L_{\text {unk }} A_{\text {ref }}
$$

Another technique that is similar in principle to the comparison technique, is what is known as the scaled subtraction technique (6). If there are spectral features present due to interfering species, the scaled subtraction technique is particularly useful. Even though this technique can be used for overlapping spectral features, either the target compound or the interfering species should have at least one unique absorption band. In this technique, the reference absorption spectrum is adjusted by a scaling factor until subtraction from the analytical absorption spectrum causes the absorption maximum of the unique band of interest to be zero. The software package used for the subtraction then reports the scaling factor which is multiplied by the concentration used to generate 
the reference spectrum to obtain the concentration of the desired analyte.

A third analytical technique is referred to as multi component analysis which can be used when multiple compounds need to be analyzed and there are multiple interfering species present (6). This scenario is not uncommon in openpath FTIR monitoring, so some type of multi component analysis is generally the preferred analytical technique. One of the most common multi component analysis methods is the classical least squares (CLS) fit. Using Iinear regression is probably more suitable than either the comparison or scaled subtraction techniques because the shape of the analytical spectrum is compared with the shape of the reference spectrum, and, a correlation coefficient can be calculated which gives a measure of this comparison. Initial Instrument Operation

\section{Distance to Saturation}

The path length at which the detector becomes saturated is a critical piece of information that should be determined if relatively short path lengths are to be used (6). To perform this test, the retroreflector or the light source is brought closer to the detector until a negative dip appears near the detector cutoff. The negative dip is opposite of 
what would appear if absorption was taking place. The light source or retroreflector is then moved away from the transmitting telescope until this negative dip disappears from the single-beam spectrum. This distance represents the minimum path length over which it is possible to operate the instrument without making changes. Sometimes it may be necessary to lower the return intensity if short path lengths are required. This can be done by rotating the retroreflector so that it is not exactly in line with the receiving telescope. A fine mesh screen can also be used to lower the intensity if necessary. It should be noted that the screen is to be constructed of wire and not plastic, because plastics have absorption features in the infrared. Return Intensity as a Function of Distance

Before being transmitted along the path, some attempt is made to collimate the infrared beam (6). Because of the size of the light source, it is impossible for the beam to be completely collimated, therefore, most beams are either diverging as they traverse the path, or they become diverging at some point along the path. The return signal will diminish as the square of the distance once the beam is bigger than the retroreflector or the receiving telescope. For example, beyond this given distance, the signal is 
reduced by a factor of four when the path length is doubled. This return signal versus distance determination is important because instruments that use the monostatic configuration have a stray light signal. Also, this determination is important because it defines the point at which the noise (including noise caused by the stray light signal) is a significant part of the signal, which is then chosen to be the maximum usable path length.

The return signal as a function of distance is determined by the retroreflector or light source beyond the minimum path distance from the detector (6). The retroreflector or light source is then moved some given distance away from the detector and the signal is recorded. This process is continued until the signal reaches the noise level.

\section{Determination of the Random Noise of the System}

An absorption spectrum made from two single-beam spectra taken sequentially is used to estimate the random noise of the system (6). The same acquisition time and path length should be used for the noise determination as will be used for the data acquisition. Only a minimum amount of time should elapse between the acquisition of the two 
spectra so that changes in concentrations of absorbing species will be minimized.

The absorption spectrum is calculated by designating one of the two single-beam spectra as the sample spectrum, and the other as a background spectrum (6). Three specific ranges are used for determining the noise: $968-1008 \mathrm{~cm}^{-1}$, 2480-2520 $\mathrm{cm}^{-1}$, and 4380-4420 $\mathrm{cm}^{-1}$. The noise can be determined from the peak-to-peak absorbances or the rootmean square (RMS) absorbance in the absorption spectrum. Peak-to-peak noise is determined by finding the minimum and maximum values of the absorbance and then subtracting the minimum from the maximum. The RMS noise is the value of the peak-to-peak noise divided by 2.8 .

\section{Return Intensity as a Function of Water Vapor}

The return beam intensity is not only a function of the path length, but also the concentration of water vapor in the atmosphere (15). Thus, water vapor concentrations should be documented when the distance to saturation, maximum usable path length, and instrument noise are estimated. A measurement of the partial pressure of water vapor in torr should be used for this purpose, not the relative humidity. Field studies have shown that there are usually no appreciable changes in the water vapor 
concentration along the path, so the measurements can be made an any arbitrary point. However, there do seem to be temporal changes in the water vapor concentration which means that water vapor concentration may need to be measured over the course of the sampling period to determine when conditions have changed.

\section{Background Spectra}

The current methods for open-path FTIR monitoring do not include recording a reference or background spectrum simultaneously with the sample spectrum to remove the spectral features due to the characteristics of the source, beam splitter, detector, and interfering species in the atmosphere (6). The ideal background spectrum would be one taken in the field under the same experimental conditions as those for the sample spectrum but without the target gas present. In many cases this is impossible to do because the target gas cannot be removed from the atmosphere.

Developing a background spectrum is probably one of the more difficult tasks in OP-FTIR monitoring, and there is no one universal method for performing this task. For this reason, the method for generating the background spectrum should be chosen on a case-by-case basis. 
If environmental monitoring is being done, a background spectrum can be acquired along a path that is upwind from the source (6). Another method is to chose a path along the side of the source, as long as the wind is not blowing across the source area which would transport the emissions across the path. One drawback to acquiring background spectra along the side of the source is that it is difficult to make these measurements frequently because the instrument has to be moved from one side of the source to another.

This lack of frequent measurements could cause errors if the amount of water vapor in the atmosphere changes

significantly between the times that the background spectrum and the sample spectra are generated. For this reason, measurements of the partial pressure water vapor in the air should again be taken frequently to determine when a new background spectrum should be taken.

If experimental conditions are fairly constant over a measurement period, it is possible to average several background spectra and use this average spectrum for the entire data set taken in that period (6). The individual spectra making up this average spectrum should have the same noise and there should be no other errors present. Due to changing concentrations of water vapor, the length of time 
for which the background spectrum will be acceptable varies with the given situation.

The fourth and final method for generating a background spectrum is the short-, or zero-path technique (6). In this method, the retroreflector or external infrared source (depending on the configuration used) is brought in close to the receiving telescope. By doing this, the absorption caused by the target gas or gases is effectively eliminated. This will result in detector saturation for most instruments. This problem can be avoided by simply turning the retroreflector or source at an angle in order to decrease the amount of signal reaching the detector. As with any background spectrum, the short-path background may only be valid for a short period of time. There are two qualitative checks that can be done to determine its validity.

1) Visually compare the curvature of the baseline in the short-path spectrum with the curvature of the baseline in the field spectra.

2) Visually compare background with field spectra to determine whether any phase shifts have occurred.

\section{Water Vapor Spectra}

Water vapor absorption lines are present everywhere in the spectrum because there is so much water in the air (16). Even when using closed path cells, the water vapor usually 
cannot be removed from the sample before analysis because there is the possibility of altering the concentrations of the pollutants that are to be measured. Because of this, the absorption features due to water vapor must be accounted for during the analysis of the field spectrum (6). The ETG (Environmental Technologies Group) software (available with the ETG Remote Sensor) allows the operator to choose water as an interfering species so that it can be accounted for during the classical least squares analysis.

A water vapor reference spectrum is needed in the script file of the ETG software in order to account for interferences (6). A suitable water vapor spectrum can be obtained from any single-beam spectrum that contains a sufficient amount of water vapor absorption in the wave number region of interest. Sometimes spectra taken at short path lengths, or those taken on days when the humidity is very low, may not be suitable for producing water vapor spectra. Also, new water vapor spectra may have to be produced as environmental conditions change. studies at Research Triangle Park showed that water vapor partial pressures could vary from less than one torr in the winter season, to as much as twenty-eight torr during the summer months. It is up to the operator of the instrument to 
determine when new water vapor spectra are needed. There are no rules as to how often this needs to be done because of environmental variability over time. In order to make this decision, the water vapor concentration should be monitored periodically to determine when a significant change has taken place (15).

One of the main concerns when producing a water vapor spectrum is to be sure that none of target gas is present in the final spectrum, because if even if any of the target gas is present, the analysis will be in error by that amount (6). A zero-path spectrum can be used as the background for producing a water vapor reference spectrum. A field spectrum collected under the same environmental conditions as will be expected during subsequent monitoring (but with no target compound present) is then used along with the zero-path to create an absorbance spectrum which is the water vapor reference spectrum. The following list is a set of criteria for the selection of single-beam spectra that are to be used for the production of a water vapor reference spectrum.

1) The single-beam spectra selected to be used to produce the water vapor reference spectrum should be taken at the same resolution as the field spectra.

2) The water vapor spectrum should have the same or better signal-to-noise ratio as the field spectra. 
3) If possible, the water vapor concentration for these two spectra should be representative of the water vapor concentration contained in the field spectra to be analyzed.

4) Curvatures and any other special features of the baselines of these spectra should be the same as those of the field spectra baselines over the wave number regions of interest.

\section{Resolution Considerations in Long-Path, OP-FTIR Monitoring}

Several factors must be considered when choosing the optimum resolution for OP-FTIR including: the ability to distinguish between the spectral features of compounds of interest and those of ambient interfering species in the atmosphere such as water vapor and carbon dioxide; tradeoffs between resolution, IR peak absorbance, and $S / N$ ratio; and practical considerations such as measurement time, computational time to process the interferogram, and the size of the interferogram file for data storage (6). If inadequate resolution is used, it can cause distortion in the absorption spectrum, affect the quantitative relationship between absorbance and concentration, and diminish the ability to resolve spectral overlap.

\section{Trading Rules in FTIR Spectroscopy}

The relationships between the signal-to-noise ratio, resolution, and measurement time in the instrument are all referred to as "trading rules". There are several factors 
that affect the signal-to-noise ratio and dictate the trading rules for a rapid-scanning Michelson interferometer (6). Signal-to-noise ratio $(S / N)$ is given by the following equation.

$$
S / N=U_{v}(T) \theta \Delta v t^{1 / 2} \xi D^{*} /\left(A_{D}\right)^{1 / 2}
$$

The term $U_{v}(T)$ is the spectral energy density at wave number $v$ from a blackbody source at a temperature $T ; \theta$ is the optical throughput which is defined as the product of the area $A$ of an aperture and the solid angle $\Omega$ of the light beam at that aperture; $\Delta v$ is the resolution of the interferometer; $t$ is the measurement time in seconds; $\xi$ is the efficiency of the interferometer; $D^{*}$ is the specific detectivity which is a measure of detector sensitivity; and $A_{D}$ is the area of the detector element.

Equation 7 shows that the signal-to-noise ratio is proportional to the square root of the total scan time $t^{1 / 2}$. Usually in FTIR monitoring, measurements are made with a rapid scanning interferometer operating at a constant mirror velocity at a given resolution (6). When this is the case, the signal-to-noise ratio increases with the square root of the number of scans being averaged. However, there are other factors that also have an affect on the signal-tonoise ratio. If physical parameters such as scanning time, 
optical throughput, and interferometer efficiency are assumed to be constant for measurements made at both high and low resolution, the signal-to-noise ratio will be halved by doubling $\Delta_{\max }$ (the maximum optical path difference of the two mirrors in a Michelson interferometer), or halving the resolution $(\Delta \nu / 2)$. As stated earlier, the signal-to-noise ratio is proportional to $t^{1 / 2}$, so the scanning time required to maintain the original baseline noise level must be increased by a factor of four each time $\Delta_{\max }$ is doubled, or when $\Delta v$ is halved. This applies only to measurements made at a constant optical throughput.

The minimum separation in wave numbers $(\mathrm{cm}-1)$ of two spectral features that can just be resolved is inversely related to $\Delta_{\max }$ given in centimeters (6). For example, if the desired resolution is increased from 1 to $0.5-\mathrm{cm}^{-1}$ (a factor of two), the moving mirror in the interferometer must travel twice as far, and therefore the scan time will be approximately twice as long. Also, the interferogram file will be approximately twice the size for data storage, and the time required to process the interferogram will be about twice as long.

Generally, the lowest possible resolution (i.e., the highest $\Delta v$ ) that adequately resolves the spectral features 
of the analyte from interfering species should be used when determining minimum detection limits (6). As stated earlier, using inadequate resolution can have several adverse effects. Likewise, using a resolution that is higher than required (i.e., $\Delta v$ lower than required) can result in a decreased signal-to-noise ratio and an unnecessary increase in measurement time, processing time, and data storage requirements. As a rule of thumb, spectra should be sampled at a resolution five times the full width at half height (FWHH) of the absorption peak of interest. Overall, the required resolution will be determined by the band widths of absorbance peaks of interest, the presence of interfering species, the signal-to-noise ratio of the system, and it may vary with specific analytes and experimental conditions.

\section{Effects of Resolution on Quantitative Analysis}

In a study by Strang et al., an FTIR system equipped with a 20.25-m multipass cell was used to monitor toxic emissions from a semiconductor manufacturing process (12). The system was designed to measure part-per-billion levels of organic vapors and metal hydrides such as arsine, phosphine, and diborane. This study addressed the selection of the optimal wave number region for quantification and the effects of resolution and spectral overlap on the accuracy 
of quantitative results. Measurements were taken at different resolutions to determine the optimum balance between analysis time, data storage space, signal-to-noise ratio, accuracy of quantitative analysis using a classic least squares program, and the ability to differentiate compounds with overlapping spectral features.

Strang et al. specified four issues that must be resolved for a classic least squares analysis at a given resolution to be valid (12). These four criteria are listed below.

1) Whether the classic least squares result varies by more than fifty percent of the theoretical value

2) Whether false positives or false negatives develop as a result of degraded resolution

3) Whether the amount of error in the measurement will cause potentially toxic concentrations of the target analyte in air to be measured incorrectly

4) Whether the detection limit obtained with the classic least squares program changes as a function of resolution

The results indicated that higher resolutions were required for the metal hydrides arsine, diborane, and phosphine due to spectral overlap with other species such as carbon dioxide overlapping with phosphine and water vapor overlapping with arsine (12). The results of the study also revealed that a decrease in resolution may cause quantitative results to be less accurate. For example, 
diborane exhibited a linear relationship for all

concentrations when a resolution of $0.5 \mathrm{~cm}^{-1}$ was used. At other resolutions of 2 and $4 \mathrm{~cm}^{-1}$, measurements deviated from linearity as the concentration decreased.

The study also addressed the effect of resolution on the ability to quantify overlapping compounds by the classic least squares analysis (12). Freon 11, 13B1, and 22 in a 1:1:1 mixture of 10,1 , and 0.1 ppm respectively were analyzed at $0.5-, 2-, 4-$, and $8 \mathrm{~cm}^{-1}$ resolutions. These compounds have broad absorption bands that overlap one another. The results indicated that the compounds could be accurately quantified at each resolution. The results of this study may or may not apply to an FTIR Remote sensor because measurements were not taken across an open path. strang and Levine also determined the LODs for the same compounds mentioned in the previous study (17). It was found that for most compounds there was little difference in the LODs at different resolutions. There were two exceptions in that diborane and phosphine were difficult to measure at $8 \mathrm{~cm}^{-1}$. The peak in the region of interest for diborane had a FWHH of $7 \mathrm{~cm}^{-1}$, so the classic least squares program could not accurately quantify it at a resolution of $8 \mathrm{~cm}^{-1}$. In the case of phosphine, the peak was severely 
degraded at $8 \mathrm{~cm}^{-1}$, so even though the classic least squares program could quantify the peak, the LOD was significantly higher at this resolution in comparison to what it would be at a resolution of $0.5 \mathrm{~cm}^{-1}$.

\section{Microbial Volatile Organic Compounds (MVOCs)}

There are literally hundreds of volatile organic compounds (VOCs) that have been identified in indoor air. These Vocs arise from both biogenic and non-biogenic sources. Non-biogenic indoor voC sources include building materials, furniture, binders and resins in carpet, paints, varnishes, and personal use products (18). Biogenic indoor VoCs are often produced by fungi and bacteria (19). Ambient concentrations of biogenic voCs are usually very low in comparison to concentrations resulting from other typical indoor sources such as those listed above (20). However, the composition of biogenic emissions differs significantly from other kinds of Vocs. These differences suggest that it may be possible to use biogenic VoCs as tracers to determine the presence of biological contamination.

The mechanisms of VOC production by fungi are poorly understood (20). Fungi digest their food outside the cell by secreting enzymes into the environment. These enzymes metabolize hydrocarbons and produce various metabolites 
including soluble sugars which are used for food, complex secondary metabolites which may be toxic to other cells, and VoCs. The exact factors that control the composition and concentration of these vocs are unknown, but the highest production of VOCs appears to precede the production of the secondary metabolites as well as the peak spore production period (21).

\section{Related Studies}

There is relatively little research information regarding specific Vocs produced by fungi, and most of the information that is available is found in food-related literature (20). Typically these studies involve microbial contamination of cereal grains. The substrate is usually placed in a clear, air-tight chamber where the organisms are allowed to grow. The headspace in the container is then sampled and analyzed to determine VOC composition. In most cases, gas chromatography with mass spectrometric detection has been the preferred method of analysis. Even though the composition of VOCs revealed by laboratory studies may differ from the composition that would actually be present in the environment, they do give us some indication of what VoCs are most commonly produced by fungi. 
Studies by Borjesson et al. report that one of the most common volatile fungal metabolites found in grains is 3 methylfuran (22). 3-Methylfuran was found to be produced by all of the storage molds examined in this study during growth on grain, but was absent in all uninoculated controls. This further confirmed that the 3-methylfuran was produced by the growing microorganisms. 3-Methylfuran has been observed to be produced by Aspergillus versicolor, which was found to be one of the most common fungal agents found in fiberglass duct Iiner samples taken from various buildings in which occupants complained of moldy air (19). In addition 3-methylfuran has a very low affinity of adsorption to materials, and thus, it can be used as an indicator of ongoing microbial growth (23).

Typically, there are marked differences in the production of volatile metabolites between closely related species and even between strains of the same fungus (22). The production of volatile metabolites can also be influenced by substrate composition, duration, and stage of fungal growth. Results of the study by Borjesson et al. revealed that this was not the case with 3-methylfuran. There was little variation in the production levels of 3 methylfuran in relation to the fungal species cultured, or 
the grain type used as the substrate. All other volatile metabolites detected showed large differences in production levels among fungal species, indicating that these compounds may be less suitable to use as indicators of general mold growth.

In the study by Borjesson et al., several criteria for determining the validity of using volatile metabolites as indicators of fungal growth were demonstrated (22). The results from the study indicated the following:

1) 3-Methylfuran was present for all fungal species tested and therefore can be used as a general indicator of fungal growth.

2) Differences in the type and concentration of vocs produced among fungal species are larger than differences related to the type of substrate.

3) The duration of fungal growth does not influence the profile of the volatile metabolites produced.

4) There was a weak correlation between $\mathrm{CO}_{2}$ production and total production of volatile metabolites. The production of volatile metabolites was more strongly correlated with accumulated production of $\mathrm{CO}_{2}$ than with actual $\mathrm{CO}_{2}$ production.

In a study by Kaminski et al. involving Aspergillus Flavus, 1-octen-3-ol was found to be one of the more predominant volatile compounds produced (24). 1-Octen-3-ol has a mushroom-like odor and is thought to be partially responsible for the characteristic musty odor of certain fungi. In the study by Kaminski et al., infrared analysis was one of the analysis techniques used. A comparison of IR 
spectra of 1-octen-3-ol isolated form $A$. Flavus with a synthetic preparation revealed that the compounds from the two different sources were identical. Although traditional infrared techniques were used, this does give hope to the possibility of using open-path FT-IR methods. The results of this study demonstrated that in general, the occurrence of 1-octen-3-ol in agricultural products can indicate their contamination by molds. Thus in theory, 1-octen-3-ol could be used as an indicator of contamination in buildings.

Information from all of the above food-related studies has been applied to the use of MVOCs as indicators of microbial contamination in buildings. In a study by McJilton et al., VoCs were used to determine what types of contamination were present (25). They discovered that vocs collected in the aix samples from the buildings were also found in a pure culture of bacterium isolated from the HVAC system. The presence of these VoCs in the buildings seemed to correlate well with the presence of symptom complaints. Other studies have shown that MVOC analysis is a useful tool to describe indoor air quality in problem houses in Germany and Sweden (23). The concentrations of MVOCs from fungi were found to be much higher in these problem houses as compared to the outdoor air and houses with no complaints. 
The study also found that different MVOC profiles correlated with the type of microorganism, growth activity, and the physical conditions of the building structure such as the amount of moisture present.

In a study by strom et al., GC-MS was used to measure to concentrations of thirteen different MVOCs, including 3methylfuran and 1-octen-3-ol (26). The samples were taken from two houses with residents suffering from indoor environmental complaints including odor problems and eye, nose, and throat irritations. The concentrations of the thirteen different compounds were added together to give a total sum of MVOCs present. This value was used to describe the condition of the indoor air. Both houses contained elevated levels of MVOCs which correlated well with microbial activity present in the construction material of these houses. However, in both cases, the concentrations ranged from 0.002 to $0.007 \mathrm{ppb}$ for 3 -methylfuran, and concentrations of 1 -octen-3-ol ranged from 0.16 to $1.9 \mathrm{ppb}$. This suggests that if these two compounds alone are to be used to describe the quality of indoor air, a very sensitive analytical technique will be required. 


\section{OBJECTIVE}

The objective of this study was to determine the feasibility of using an FTIR Remote sensor for monitoring two MVOCs in indoor environments for the purpose of determining whether or not microbial contamination is present. Based on the current literature, 3-methylfuran and 1-octen-3-ol can be used as indicators of fungal growth in contaminated buildings. For the purpose of this study, feasibility was based on the lower detection limits for these two compounds. The current literature suggests that the expected concentrations of 3-methylfuran and 1-octen-3ol in contaminated buildings is less than 0.007 and $1.9 \mathrm{ppb}$ respectively. In order to determine that the use of OP-FTIR is feasible for this particular application, LODs less than these values had to be achieved for each compound. 


\section{MATERIALS AND METHODS}

\section{Description of the Instrument}

An ETG (Environmental Technology Group) Model 282000 Open-Path FTIR Remote Sensor was used for all experiments. The instrument is equipped with a Model 282302 MCT detector cooled by a Stirling engine cryocooler. The standard system includes a continuous scan Michelson interferometer which has a spectral range of $700 \mathrm{~cm}^{-1}$ to $5000 \mathrm{~cm}^{-1}$, has dimensions of $12.25 " \mathrm{x} 18.5 " \mathrm{x} 24.25 "$, and an operating temperature range of 0 to $40^{\circ} \mathrm{C}\left(32\right.$ to $\left.104^{\circ} \mathrm{F}\right)$. The transmitting and reflecting optics include a Model 282074 Cassegrain telescope which produces an IR beam divergence of 2.2 milliradians, and a Model 282615 retroreflector which consists of a 30-cube retro array protected with a gold front surface with dimensions of 17"x 17"(max). Each cube has a diameter of $2.5 "$ and is accurate to 30 arc seconds. The resolution of the instrument was set at $1.0 \mathrm{~cm}^{-1}$ for all experiments because both compounds have low absorptivity coefficients. 


\section{Development of Reference Spectra}

In order to develop infrared reference spectra for 3methylfuran and 1-octen-3-ol, a multipass cell was needed so that the spectra could be taken in the absence of any interferants. Southern Research Institute (SORI) located at Research Triangle Park uses such a cell along with an ETG instrument that is identical to the one used in this study. For this reason, SoRI was employed to aid in the generation of the reference spectra because the spectra must be of high quality in order for quantification of the gases to be accurate.

Compressed gas cylinders of 3-methylfuran and 1-octen3-ol were obtained from Scott Specialty Gases (Durham, NC) at concentrations of $10.36 \mathrm{ppm}$ and $10.3 \mathrm{ppm}$ respectively in nitrogen. An Infrared Laboratories heatable multipass cell (Model OVG-5-22-H-BA-AU) was connected to the ETG instrument via the transfer optics. Laboratory conditions were kept as close as possible to the standard conditions defined by the ETG analysis software $(T=296 \mathrm{~K}$ and $\mathrm{P}=760$ torr $)$. The path length of the cell was set at 17.72 meters and verified by analyzing a known concentration of ethylene obtained from Scott Specialty Gases. 
Brass regulators were fitted to the cylinders containing the 3-methylfuran and 1-octen-3-ol. The gases were introduced directly into the cell through a short length of $1 / 4$ inch Teflon tubing connected with Swagelok fittings. Prior to the introduction of a gas, the cell was evacuated to less than $10^{-2}$ torr using a double stage rotary pump. The cell was purged with "zero" air obtained from Scott Specialty Gases. Background spectra of 64 co-added scans were recorded while the cell was filled with the zero air.

Each analyte was introduced into the cell separately. The gases were continuously flowed through the cell in order to minimize any losses due to adsorption of the gases onto the Teflon coated walls of the cell. The flow rates used were between 200 and $300 \mathrm{~cm}^{3}$ per minute. Reference spectra of 32 co-added scans were recorded for each of the analytes. Corrections for slight differences in temperature and pressure from that specified in the ETG software were made. The regions for analysis were chosen based on the intensity of the spectrum and the expected overlap with common interferants found in open path measurements (e.g., $\mathrm{H}_{2} \mathrm{O}$ ). The multipass cell at SORI was used to determine detection limits that can be achieved in the absence of any 
interferants. The LOD for each compound was determined by introducing the gases into the cell, then diluting the gases continuously with zero air. Successive measurements were recorded using the ETG continuous monitoring software until the concentration reached a level near three standard deviations above the RMS noise. At this point, the absorbance of the target compound is no longer significantly different than the baseline of the spectrum. This concentration is considered to be the LOD for that particular compound under the environmental conditions of the test.

\section{Field Tests}

The instrument was taken into the field in order to determine the effect of varying the path length and scan time on detection limits. Data were collected at path lengths of 10,50 , and 100 meters, and $128,64,32,16,8$, 4, 2, and 1 co-added scans. The measurements were taken in an indoor environment at the UNC School of Public Health so as to simulate the response of the instrument in conditions that would be expected in typical buildings. The instrument was set up inside the building facing down a long hall.

The retroreflector was first placed 5 meters in front of the instrument. The distance was measured from the end 
of the telescope to the front of the retroreflector. The monostatic configuration was used, so placing the retroreflector at a distance of 5 meters gave a total path length of 10 meters. The beam was aligned with the retroreflector which resulted in detector saturation due to the short path length. The beam was slightly turned away from the retroreflector in order to decrease the signal strength to an acceptable level of approximately 5000 signal intensity units.

Six spectra of 128 co-added scans were recorded at a path length of 10 meters, followed by six spectra at 64 and 32 co-added scans. Twelve spectra of 16 co-added scans were recorded at the same path lengih, followed by twelve spectra at $8,4,2$, and 1 co-added scans. This process was repeated at path lengths of 50 and 100 meters, meaning the retroreflector was placed at distances of 25 and 50 meters away, respectively.

Two zero path spectra (retro directly in front of telescope) of 128 co-added scans were recorded. The retroreflector was turned slightly away from the instrument to avoid detector saturation. The retroreflector was turned away until a signal strength was decreased to approximately 
5000 signal intensity units. This same procedure was conducted at each new path length.

The temperature and relative humidity were monitored at about one hour intervals when data was being collected. A Model 11-661-7B certified traceable digital hygrometer/thermometer manufactured by Fisher scientific was used to take these measurements. These measurements were used to make sure water vapor concentrations did not drastically change over the time period of data collection. Detection limits for 3-methylfuran and 1-octen-3-ol were calculated using the available ETG software which performs a least squares analysis between the observed spectrum and the reference spectrum in the wave number region of interest. The ETG software requires that a script file be defined for each analysis. A script file contains information about the target compound and possible interferants. The operator designates the compounds of interest and interferants, tells the computer exactly where the reference spectra for each of theses compounds are located in the spectral library, and specifies the wave number ranges for the regions of interest. Script files for each compound were generated by entering the appropriate information. Water was chosen as the primary interferant. 
Multiple water vapor spectra were produced for this purpose by using a zero path spectrum as a background, and selecting one spectrum for each scan time at each path length as a data spectrum. The absorbance spectra produced from these single beam spectra were used as the water vapor reference spectra.

Absorbance spectra were created from the single beam spectra by choosing the first spectrum taken for a particular number of scans and path length as a background, and using the subsequently acquired spectra taken at that same number of scans and path length as data spectra. The backgrounds used for a particular set of data were always produced at the same path length and with the same number of scans so that the backgrounds and data spectra would have as close to the same features as possible. This was done so as to reduce the noise in the absorbance spectra as much as possible. LODs were calculated for each absorbance spectra created. The mean of the LODs for each spectrum taken at the same scan time and path length was used for the reported result.

The water vapor concentration of the spectra taken at UNC was adjusted in order to determine how the detection limits would be affected. The water vapor concentration was 
synthetically altered using the Labcalc software package (available from ETG) which allows for the addition or subtraction of peaks areas in a particular spectrum. The original data was taken at a relative humidity of $62 \%$ and a temperature of $22^{\circ} \mathrm{C}$, which corresponds to a water vapor pressure of approximately 12.5 torr. The concentration of water was computationally decreased to a water vapor pressure of approximately 7 torr $(\mathrm{RH}=34 \%)$, and was increased to a water vapor pressure of approximately 16 torr $(\mathrm{RH}=77 \%)$. The alterations were performed on each single beam spectrum taken, including spectra considered as backgrounds and data spectra. The single beam spectra were altered rather than the absorbance spectra, so as to simulate the results that would be obtained if the entire study had been done on a day of higher or lower absolute humidity, meaning that both backgrounds and data files would contain approximately the same concentration of water. This was done in order to determine if noise created by differences in background and data spectra would be more or less pronounced at either higher or lower concentrations of water vapor. The same procedures used to collect the original data for producing absorbance spectra and 
calculating LODs were used so that the results could be compared to the original data.

\section{Statistical Analysis}

The limit of detection (LOD) can be defined as the lowest concentration level that can be determined to be statistically different from an analytical blank (27). The term "statistically different" can be defined in many different ways. The International Union of Pure and Applied Chemists (IUPAC), as well as many others, define the term "statistically different" as being a response that exceeds the instrument noise level by three standard deviations above the mean of the instrument noise. Likewise, the limit of quantitation (LOQ) is often defined as the concentration of analyte that provides a detector response that exceeds the instrument noise level by ten standard deviations above the mean of the instrument noise. A study by strang and Levine revealed that the LOD values calculated by a least squares fit method may provide a better estimation of the performance characteristics of a FTIR instrument than the LODs based solely on a $\mathrm{S} / \mathrm{N}$ of three standard deviations (17)

In order to apply any least squares method to quantitative infrared measurements, there must be a known 
relationship between the infrared spectrum and the quantity of the analyte present (see Eq. 3)(28). The Beer-Lambert relationship states that the concentration is directly proportional to the absorbance ( $A$ ) and is given by

$$
A=\alpha C L
$$

where $\alpha$ is the absorptivity and $L$ is the path length at a given wavelength.

A least squares fit of environmental to reference spectra corresponds to the application of Beer's law to the entire spectrum of wavelengths in the region of interest. This is a primary advantage over use of the Beer-Lambert relationship at one wavelength because most of the spectral information contained in the sample spectrum is related by least squares methods to the reference spectrum for the particular compound of interest (28). The least squares fit yields an average response factor $(k)$ for the calculation of the sample concentration $\left(C_{s}\right)$ from the concentration of the reference sample $\left(C_{r}\right)$ by applying the following relationship:

$$
C_{s}=k C_{r}
$$

There are currently four different least squares methods that have been devised and tested by Haaland and Easterling (28). The differences in the four approaches are 
based on the following four assumptions made about the baseline of the sample spectrum: (1) baseline is zero or is accurately known and can be fitted to zero, (2) the baseline is linear over the spectral region of interest, (3) the baseline is linear over each spectral peak, and (4) the baseline is essentially constant between successive data points.

Theoretically, the improvement in defining the LOD precisely generally decreases as the assumptions about the baseline become less restrictive, that is, method (1) would show the greatest precision and (4) would show the least (28). However, the assumption of the first method that the baseline is accurately known is rarely the case, so method (1) is not usually the preferred method.

The ETG software uses the assumption of a fixed and linear baseline over the spectral range of interest in the reference and sample spectra. This assumption is described by Haaland and Easterling as method 2 (28). The least squares analysis uses all the spectral data above a selected absorbance threshold in the spectral region of interest. In the ETG software, this absorbance threshold is set at 3,6 , or 9 standard deviations above the RMS noise. The least squares analysis performs a fit of the spectral baselines 
and a quantitative determination of each component specified in the script file as a compound of interest. 


\section{RESULTS AND DISCUSSION}

\section{Results}

\section{Reference Spectra}

Reference spectra for 3-methylfuran and 1-octen-3-ol were generated in the absence of any interferants. The path length of the cell was 17.72 meters. The concentration of 3-methylfuran as reported by Scott Specialty Gases was 10.36, giving a path integrated concentration of $183.6 \mathrm{ppm}-\mathrm{m}$ at which the reference spectrum was generated. The concentration of 1-octen-3-ol was reported as $10.3 \mathrm{ppm}$, giving a path integrated concentration of $182.5 \mathrm{ppm}-\mathrm{m}$. The reference spectra (Figures 6 and 7 ) show that both compounds are fairly weak absorbers in the infrared region. The regions of the spectra with the highest intensity were between 741.01 and $807.06 \mathrm{~cm}^{-1}$ for 3 -methylfuran (shown in Figure 8), and between 909.27 and $944.46 \mathrm{~cm}^{-1}$ for 1 -octen-3ol (shown in Figure 9). In these regions, 3-methylfuran and 1-octen-3-ol had absorptivity coefficients of 0.157 and $0.0215 \mathrm{~m}^{-1} \mathrm{ppm}^{-1}$ respectively. 


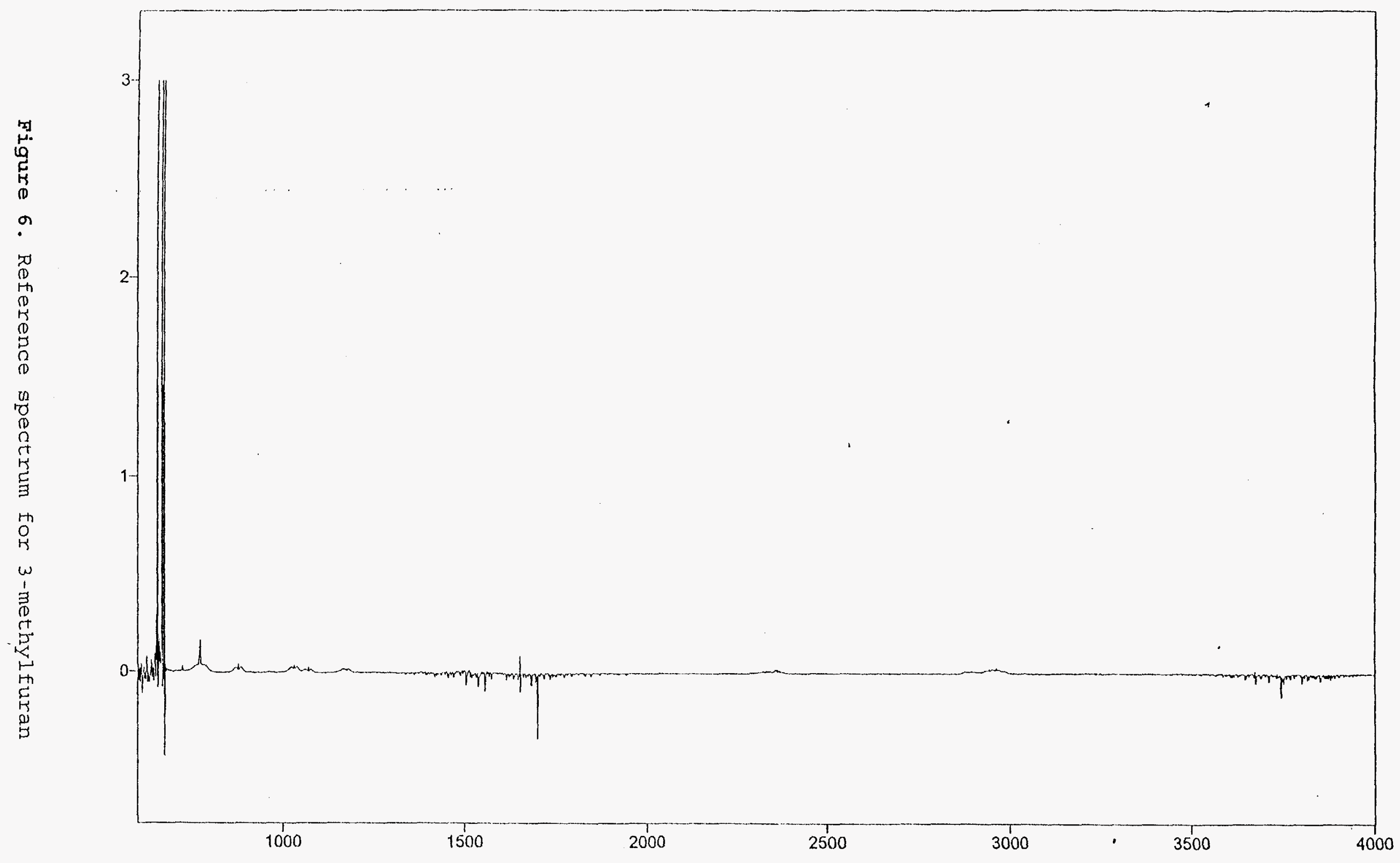

Absorbance / Wavenumber (cm-1)

Paged Y-Zoom CURSOR

File \#1 = A1MRT024

8/3/95 8:40 AM Res=None 


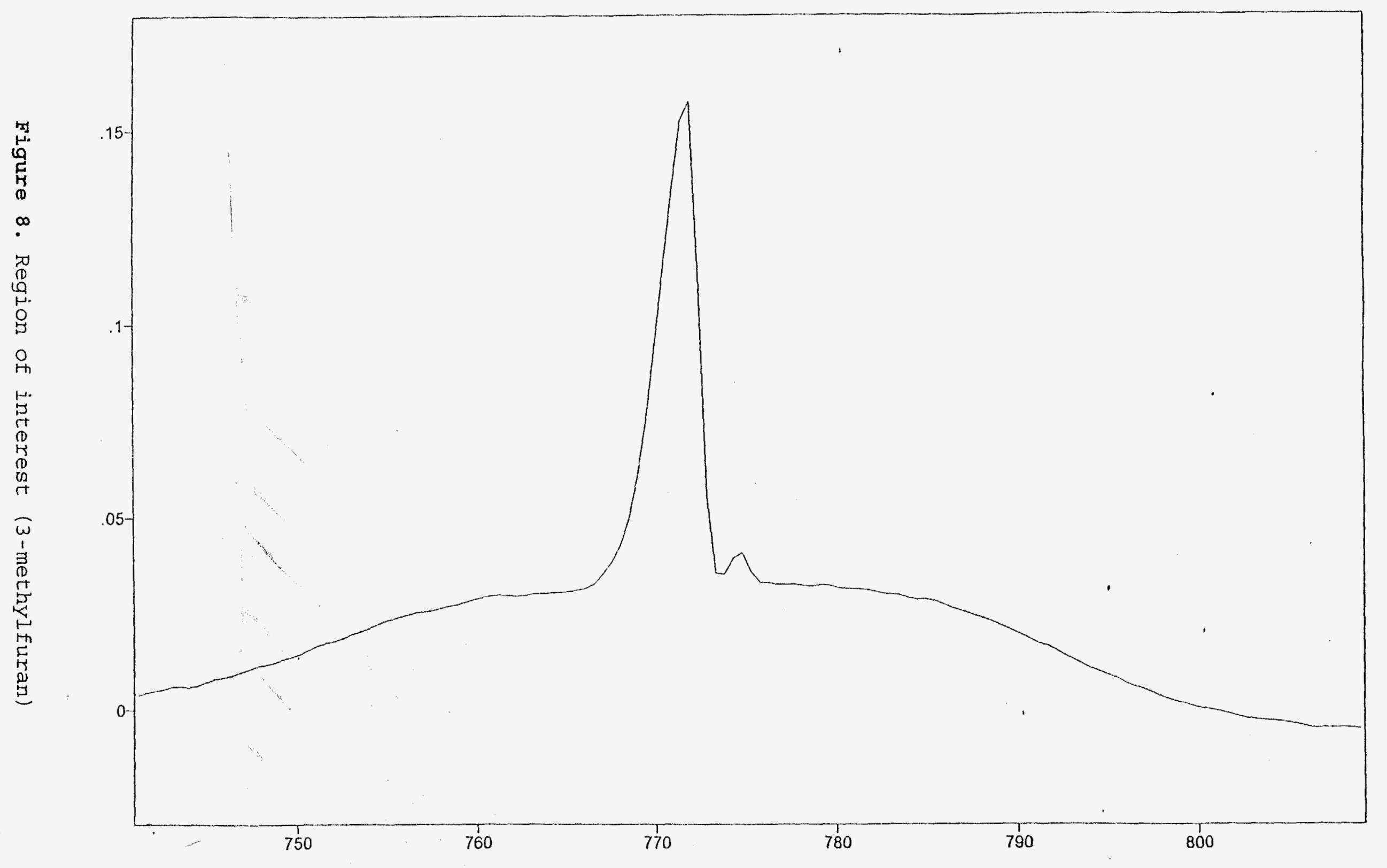

Absorbance / Wavenumber (cm-1)

Paged Y-Zoom CURSOR

File \# $1=$ A1MRT024

8/3/95 8:40 AM Res=None

10.36ppm 3-methyl-furan $17.72 \mathrm{mpl} 293.4 \mathrm{k} 756.9 \mathrm{mmHg}$ 


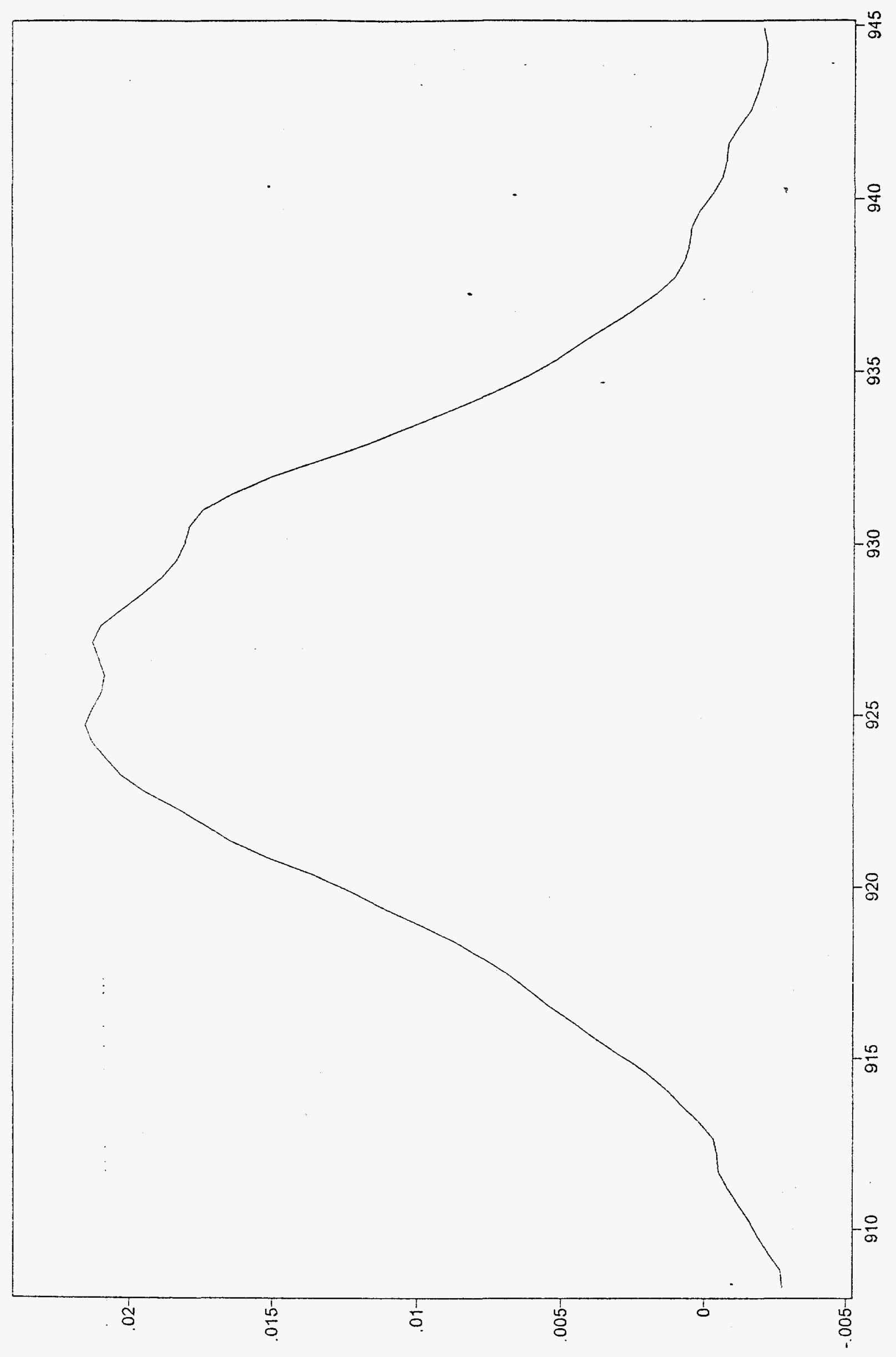

Figure 9. Region of interest (1-octen-3-ol)

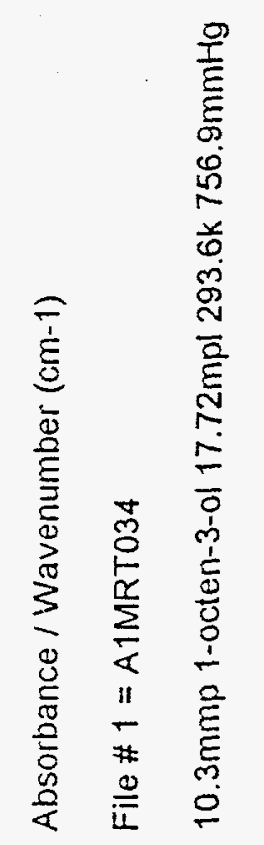




\section{Limits of Detection}

The LODs for both compounds for 128 scans at a temperature of $22^{\circ} \mathrm{C}$ and a water vapor pressure of 12.5 torr are shown in Table I. The LODs Iisted are considered to be for a best case scenario, meaning the backgrounds were taken immediately preceding the data spectra. The LOD reported for each path length is the mean of the LODs reported for five spectra taken at that path length. A 95\% confidence interval is reported along with each LOD. Results of LODs calculated for other scan times are given in Appendix A.

Table I. Summary of LODs for Original Data at $22^{\circ} \mathrm{C}$ and a Water Vapor Pressure of 12.5 torr

\begin{tabular}{|c|c|c|c|c|c|c|c|}
\hline \multicolumn{4}{|c|}{ LODs calculated for 3-methylfuran (ppb) } & \multicolumn{4}{|c|}{ LODs calculated for 1-octen-3-ol (ppb) } \\
\hline & $10 \mathrm{~m}$ & $50 \mathrm{~m}$ & $100 \mathrm{~m}$ & & $10 \mathrm{~m}$ & $50 \mathrm{~m}$ & $100 \mathrm{~m}$ \\
\hline Mean & 148 & 11 & 8 & Mean & 447 & 34 & 23 \\
\hline $95 \%$ C.I. & $(132-164)$ & $(8-14)$ & $(6-10)$ & $95 \%$ C.I. & $(306-588)$ & $(28-40)$ & $(22-23)$ \\
\hline
\end{tabular}

The mean LODs for each compound were affected by varying the path length and the number of scans. In general, the LOD initially decreased as path length and number of scans increased and usually leveled out. This general trend is shown for both 3-methylfuran and 1-octen-3ol in Figures 10 and 11. 


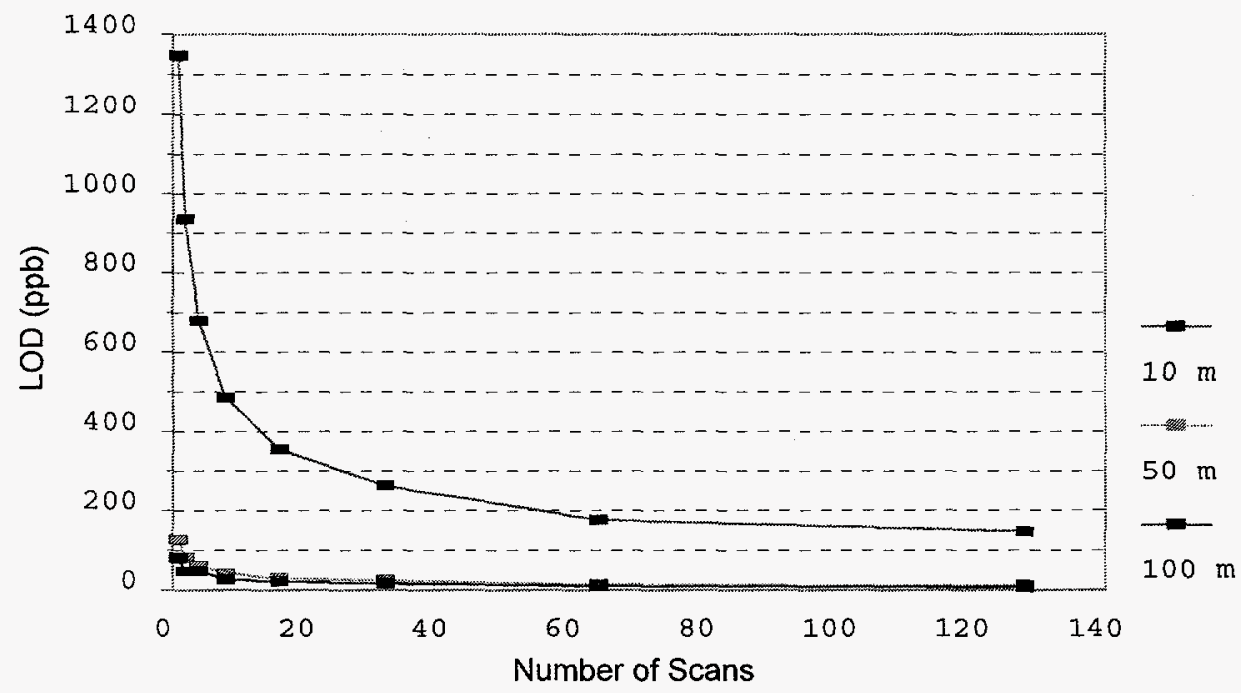

Figure 10. Affects of Scan Time and Path Length on LODs (3-methylfuran)

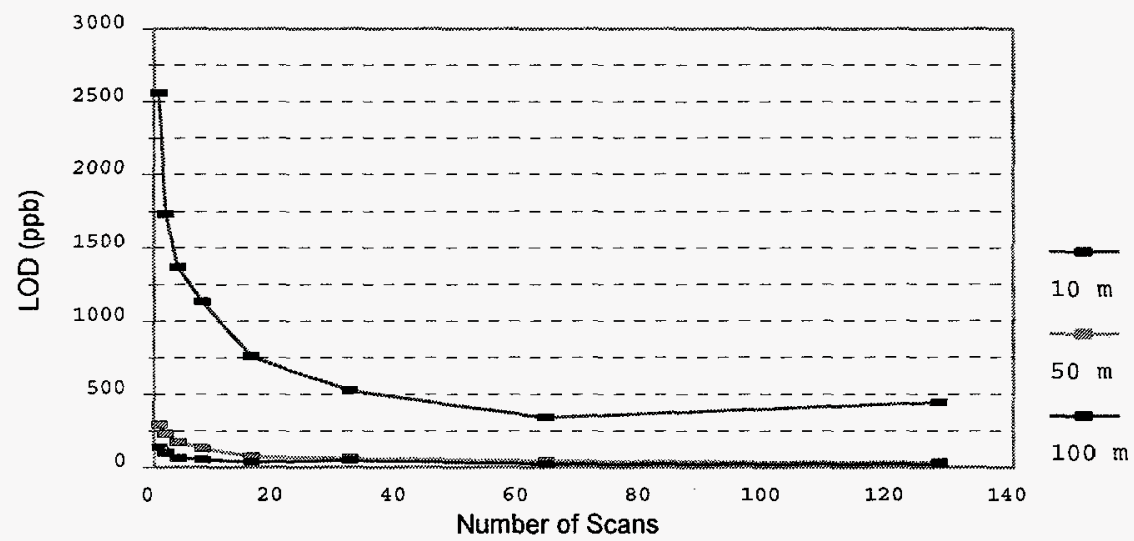

Figure 11. Affects of Scan Time and Path Length on LODs (1-octen-3-ol) 
LODs for 128 scans calculated for data at a simulated relative humidity of $34 \%$ are presented in Table II. The LODs were calculated using the same methods as for the original data, only at a lower concentration of water vapor. Results of LODs calculated at other scan times are given in Appendix A.

Table II. Summary of LODs for $34 \% \mathrm{RH}$ Data

\begin{tabular}{|c|c|c|c|c|c|c|c|}
\hline \multicolumn{4}{|c|}{ LODs calculated for 3-methylfuran (ppb) } & \multicolumn{4}{|c|}{ LODs calculated for 1 -octen-3-ol (ppb) } \\
\hline & $10 \mathrm{~m}$ & $50 \mathrm{~m}$ & $100 \mathrm{~m}$ & & $10 \mathrm{~m}$ & $50 \mathrm{~m}$ & $100 \mathrm{~m}$ \\
\hline Mean & 132 & 13 & 7 & Mean & 439 & 34 & 22 \\
\hline $95 \%$ C.I. & $(119-145)$ & $(7-18)$ & $(6-9)$ & $95 \%$ C.I. & $(291-586)$ & $(28-41)$ & $(22-23)$ \\
\hline
\end{tabular}

LODs calculated for data at $77 \%$ relative humidity are presented in Table III. The LODs were calculated using the same methods as for the original data, only at a higher concentration of water vapor. Results of LODs at other scan times are given in Appendix A.

Table III. Summary of LODs for $77 \%$ RH Data

\begin{tabular}{|l|c|c|c|l|c|c|c|}
\hline \multicolumn{2}{|c|}{ LODs calculated for 3-methylfuran (ppb) } & \multicolumn{5}{c|}{ LODs calculated for 1-octen-3-ol (ppb) } \\
& $10 \mathrm{~m}$ & $50 \mathrm{~m}$ & $100 \mathrm{~m}$ & & $10 \mathrm{~m}$ & $50 \mathrm{~m}$ & $100 \mathrm{~m}$ \\
\hline Mean & 339 & 23 & 278 & Mean & 449 & 34 & 22 \\
\hline $95 \%$ C.I. & $(211-467)$ & $(13-33)$ & $((-48)-603)$ & $95 \%$ C.I. & $(298-600)$ & $(27-40)$ & $(22-23)$ \\
\hline
\end{tabular}


The LODs determined in the experiments conducted at SoRI for 3-methylfuran and 1-octen-3-ol were 150 and 210 ppb respectively, at a path length of 17.72 meters, and using 32 co-averaged scans.

Appendix B contains examples of several different spectra collected in this study including water vapor reference, zero-path, single-beam, and absorbance spectra. Discussion

Based on the results of the study conducted by strom et al., the concentrations of 3-methylfuran and 1-octen-3-ol that would typically be found in a microbial contaminated indoor environment would be less than 0.007 and $1.9 \mathrm{ppb}$ respectively (26). In order for open path FTIR to be used for monitoring MVOCs, LODs less than these values would be required for the two individual compounds. The results of this study showed that LODs lower than the values given above were not achieved. Table I shows the lowest LOD achieved which was 8 ppb for 3-methylfuran when a path length of 100 meters was used. The LOD would be expected to be lowest at the longest path length because more absorption of the gas takes place along the path. This will hold true until the path length becomes so long that the stray light signal becomes a significant interference. Table I also 
shows that the LOD for 1-octen-3-ol was higher under the same given set of circumstances, which is due to its lower absorptivity coefficient.

Table I shows that generally, as path length increases, the LOD will decrease with the number of scans being held constant. The mean values at $50 \mathrm{~m}$ and $100 \mathrm{~m}$ for 3 -methylfuran were not significantly different, but the data for 1-octen3-ol are consistent with this general trend, except for a increase in the LOD in the $10 \mathrm{~m}$ data when the number of scans was increased from 64 to 128. This discrepancy may be due to a lack of warm-up time for the instrument. When the $10 \mathrm{~m}$ data was collected, the instrument had only been on for a few minutes, whereas when the other data was collected, the instrument had been on for several hours. Figures 10 and 11 also show the general trend when both path length and scan time are increasing. This is consistent with what would be expected in that over longer paths, the gas absorption is greater, and using longer scan times averages the noise in the spectra, both of which would allow for lower LODs. There is a limit as to how low the LOD can be driven by using a larger number of scans. The more scans are coadded, the more the noise is averaged, and therefore lowered. However, it does seem that there is a definite 
limit as to how long this averaging affect is advantageous. During this study, data was collected using more than 128 co-added scans to see if the LoDs could be lowered. It was found that in general, the LODs at these higher scan times were not significantly different than those obtained at 128 scans. Therefore, it would be a disadvantage to use more than 128 scans because an unnecessary averaging of data would be taking place. That is, the instrument begins to become more of an integrated sampler rather than a real-time instrument.

Tables II and III show the LODs calculated when the water vapor concentration was altered. Once again, these LODs are calculated from 128-scan data. In general, the LODs calculated at lower water vapor concentrations are less than those calculated for the original data. Likewise, the LODs calculated when the water vapor concentration was increased are larger relative to the original data. This only shows a general trend of what results one might see if the study had been conducted under different environmental conditions. The mean LODs for 3 -methylfuran at $34 \%$ and $77 \%$ RH were significantly different from one another only at a path length of 10 meters. The mean LODs for 1-octen-3-ol at $34 \%$ and $77 \% \mathrm{RH}$ were not significantly different from one 
another at any of the three path lengths. The mean LODs at 50 and 100 meters for 3-methylfuran at $34 \% \mathrm{RH}$ were not significantly different from one another but were significantly different from the mean LOD at 10 meters under the same conditions. Likewise, the mean LODs at all three path lengths for 3-methylfuran at $77 \% \mathrm{RH}$ were not significantly different from one another. LODs calculated at each path length for 1-octen-3-ol were significantly different from one another for each water vapor concentration.

The difference between how the two compounds are affected by water vapor can be explained by the difference in the extent of absorption due to water vapor that takes place in their respective regions chosen for analysis. The region of analysis chosen for 1-octen-3-ol contained fewer water absorption peaks than the region chosen for 3methylfuran. Because there are fewer water absorption bands, changes in water vapor concentration will not affect the results for 1 -octen-3-ol to the same extent. Therefore, LODs for 1-octen-3-ol remain more constant than LODs for 3methylfuran as water concentration changes, and there is not as much uncertainty present in the least squares fit. 
The general trend of decreasing LOD with increasing number of scans was not observed for 3-methylfuran in the $77 \% \mathrm{RH}$ data at $100 \mathrm{~m}$. The LOD actually increased at $100 \mathrm{~m}$ relative to that of $50 \mathrm{~m}$. One possible explanation for this discrepancy is the fact there is more water vapor present in this particular situation than in any other tested in this experiment. This is due to the fact that the region of interest for 3-methylfuran contains the most water absorption bands, the relative humidity is at $77 \%$, and more absorption due to water vapor takes place at $100 \mathrm{~m}$ than at shorter path lengths. This is likely the reason why the confidence interval was so large and included negative values.

The importance of when the background is taken depends on how much variability is present with regards to environmental conditions, such as humidity, temperature, and pressure. If environmental conditions are relatively stable, backgrounds are valid for a longer period of time as compared to when environmental conditions are changing. Small changes is the environment will always be present, and it is difficult to quantify the amount of change that must take place before the background is no longer valid. In this study, it was convenient and desirable to take 
backgrounds immediately preceding the data spectra in order to determine LODs in a best case scenario. By doing this, we can assume that the LODs determined in this study are the lowest that could theoretically be achieved under this given set of circumstances.

As mentioned earlier, the backgrounds used in this study were taken at a scan time equal to that of the data spectra. The raw data presented in Appendix A show that as scan time decreases, the LODs increase. This increase in the LODs is not only due to the decrease in the number of scans, but also the decrease in the number of scans used to collect the background spectra. In this study, the number of scans used to collect the sample spectra and their corresponding background spectrum were always equal, but this may not be the best method, especially when dealing with lower numbers of scans, such as 32 and less. In theory, it would be better to use backgrounds taken at scan times greater than that of thé corresponding data spectra, again, especially at small scan times. Because of the longer averaging times, the background would contain less noise, and thus, the absorbance spectrum would also contain less noise. Therefore, if data spectra were collected at 32 scans, it would be much better to use backgrounds collected 
at a larger number of scans such as 64 or 128 , rather than using 32-scan backgrounds. By using backgrounds with longer averaging times, theoretically the LODs achieved in this study could be lower, but there again, there is a limitation as to how much of the noise will be averaged out. The number of scans for the background and data spectra were made equal in this study in order to show the general

effects of increasing and decreasing the number of scans on the LOD.

It should be noted that the concentrations of the target compounds that might possibly have been present during the time the spectra were collected are negligable as compared to the concentration of water vapor in the air. Just as when the concentration of water vapor was lowered and the LODs were not greatly affected, the LODs would not significantly be affected when estimated by the procedures done in this study, even if some absorption features due to 3-methylfuran and 1-octen-3-ol were present in the spectra. 


\section{CONCLUSIONS}

The results of this study show that open path FTIR can be used to monitor for 3-methylfuran and 1-octen-3-ol in the lower ppb range. However, according to the available literature, the typical concentrations of these compounds in contaminated environments will be less than the LODs achieved in this study. Based on these findings, using open path FTIR to detect microbial contamination by monitoring 3 methylfuran and 1-octen-3-ol is marginally feasible for the general monostatic configuration. To obtain lower LODs with the currently available instruments, longer path lengths would be required. Unless the size of the room in question is relatively large, the instrument set up used in this study would not be sufficient. Theoretically, it is possible to use multiple retroreflectors in order to achieve longer path lengths. Further research in this area could possibly lead to new instrument configurations and may allow for lower LODs. Also, as the technology advances, higher resolution instruments will be made available. If 
resolutions of less than one wavenumber could be achieved, then the quantitative abilities of the instrument would greatly improve, and would allow for lower LODs. There is a great deal of research being conducted with OP-FTIR at the present, and as a result, this technology will surely be used for many different applications in the future. 


\section{Bibliography}

(1) Xiao, H-K., S.P. Levine, J.B. D'Arcy, G. Kinnes, and D. Almaguer. 1990. Comparison of the Fourier Transform Infrared (FTIR) Spectrophotometer and the Miniature Infrared Analyzer (MIRAN) for the Determination of Trichloroethylene (TCE) in the Presence of Freon 113 in Workplace Air. American Industrial Hygiene Association Journal 51(7):395-401.

(2) Hanst, P.I., N.W. Wong, and J. Bragin. 1982. A Long-Path Infra-Red Study of Los Angeles Smog. Atmospheric Environment $16(5): 969-981$.

(3) Franzblau, A., S.P. Levine, L.A. Burgess, A.S. Qu, R.M. Schreck, and J.B. D'Arcy. 1992. The Use of a Transportable Fourier Transform Infrared (FTIR) Spectrometer for the Direct Measurement of Solvents in Breath and Ambient Air -I: Methanol. American Industrial Hygiene Association Journal 53(4)221-7.

(4) Skoog, Douglas A., and James J. Leary. 1992. Principles

of Instrumental Analysis: Fourth Edition. Saunders College Publishing, New York, pp. 252-295.

(5) Hanst, P.L. 1970. Infrared Spectroscopy and Infrared 
Lasers in Air Pollution Research and Monitoring. Applied Spectroscopy $24(2): 161-174$

(6) Russwurm, George M., and Jeffrey W. Childers. 1995. FTIR Open-Path Monitoring Guidance Document: Second Edition. ManTech Environmental Technology, Inc. Research Triangle Park, North Carolina.

(7) Herget, W.F. 1982. Analysis of Gaseous Air Pollutants Using a Mobile FTIR System. American Laboratory $14(12): 72-78$

(8) Herget, W.F., and J.D. Brasher. 1979. Remote Measurement of Gaseous Pollutant Concentrations Using a Mobile Fourier Transform Interferometer system. Applied Optics $18(20): 3404-3420$.

(9) Malachowski, M.S., S.P. Levine, G. Herrin, R.C. Spear, M. Yost, and Z. Yi. 1994. Workplace and Environmental Air Contaminant concentrations Measured by Open Path Fourier Transform Infrared Spectroscopy: A Statistical Process Control Technique to Detect Changes from Normal Operating Conditions. Journal of Air \& Waste Management Association 44:673-682.

(10) Todd, L., and D. Leith. 1990. Remote Sensing and Computed Tomography in Industrial Hygiene. American 
Industrial Hygiene Association Journal 51 (4):224233.

(11) Herget, W.F. 1982. Remote and Cross-Stack Measurement of Stack Gas Concentrations Using a Mobile FT-IR System. Applied Optics 21(4):635-641.

(12) Strang, C.R., S.P. Levine, and W.F. Herget. 1989. A Preliminary Evaluation of the Fourier Transform Infrared (FTIR) Spectrometer as a Quantitative Air Monitor for Semiconductor Manufacturing Process Emissions. American Industrial Hygiene Association Journal $50(2): 70-77$.

(13) Levine, S.P., and G.M. Russwurm. 1994. Fourier Transform Infrared Optical Remote sensing for Monitoring Airborne Gas and Vapor Contaminants in the Field. Trends in Analytical Chemistry 13(7):258262 .

(14) Hanst, Philip L. 1992. Infrared Spectra for

Quantitative Analysis of Gases. SP-8I Optical Remote Sensing. Applications to Environmental and Industrial Safety Problems, Air \& Waste Management Association, Pittsburgh, PA, pp. 490-510.

(15) Luke, R., Kricks, R., and D. Pescatore. 1992. Effects of Atmospheric Water Vapor on Open-Path FTIR 
Spectroscopy Data. Blasland, Bouck, and Lee. Edison, NJ, pp. $1-9$

(16) Hanst, P.L., A.S. Lefohn, and B.W. Gay, Jr. 1973.

Detection of Atmospheric Pollutants at Parts-per-

Billion Levels by Infrared Spectroscopy. Applied Spectroscopy $27(3): 188-198$

(17) Strang, C.R., and S.P. Levine. 1989. The Limits of

Detection for the Monitoring of semiconductor Manufacturing Gas and Vapor Emissions by Fourier

Transform Infrared (FTIR) Spectroscopy. American

Industrial Hygiene Association Journal 50(2):78-84.

(18) Wallace, L., Volatile Organic Compounds in Indoor Air Pollution: A Health Perspective, Samet, J.M. and Spengler, J.D., Eds., Johns Hopkins University Press, Baltimore, MD, 1991a.

(19) Ezeonu, I.M., D.L. Price, R.B. Simmons, S.A. Crow, and D.G. Ahearn. 1994. Fungal Production of Volatiles during Growth on Fiberglass. Applied and Environmental Microbiology 60(11): 4172-4173.

(20) Burge, Harriet A. 1995. Bioaerosols. CRC Press Inc., New York, pp. 249-268.

(21) Abramson, D., Sinha, R.M., and J.T. Mills. 1983. Mycotoxins and odor Formation in Barley stored at 16 
and 20\% Moisture in Manitoba, Cereal Chemistry, $60: 350$

(22) Borjesson, T., U. Stollman, and J. Schnurer. 1992. Volatile Metabolites Produced by Six Fungal species Compared with Other Indicators of Fungal Growth on Cereal Grains. Applied and Environmental Microbiology $58(8): 2599-2605$.

(23) Wessen, B., Strom, G. and S. Schoeps, K-O. 1994. MVOC Profiles - A Tool for Indoor-Air Quality Assessment. Indoor Air-An Integrated Approach, International Workshop, Gold Coast, Australia.

(24) Kaminski, E., I.M. Libbey, S. Stawicki, and E. Wasowicz. 1972. Identification of the Predominant Volatile Compounds Produced by Aspergillus flavus. Applied Microbiology $24(5): 721-726$.

(25) McJilton, C.E., S.J. Reynolds, S.K. Streifel, and R.L. Pearson. 1990. Bacteria and Indoor Odor Problems. American Industrial Hygiene Association Journal $51: 545$

(26) Strom, G., West, J., Wessen, B. and U. Palmgreen. 1994. Health Implications of Fungi in Indoor Environments. Elsevier Science B.V., Amsterdam, pp. 291-305.

(27) Long, Gary I., and J.D. Winefordner. 1983. Limit of 
Detection: A Closer Look at the IUPAC Definition. Analytical Chemistry 55(7):712A-721A.

(28) Haaland, D.M., and R.G. Easterling. 1982. Application of New Least-squares Methods for the Quantitative Infrared Analysis of Multi component Samples. Applied Spectroscopy $36(6): 665-673$ 
Appendix A

Raw Data 


\begin{tabular}{|c|c|c|c|c|c|c|c|}
\hline \multicolumn{8}{|c|}{ MDLs calculated by ETG software: 3 -methylfuran. $10 \mathrm{~m}$ pathlength. 3 sigma values } \\
\hline & & & & & 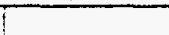 & & \\
\hline \multicolumn{8}{|c|}{ Path Integrated Data (pom-m) } \\
\hline $128 \operatorname{scans}$ & 64 scans & 32 scans & 16 scans & 8 scans & 4 scans & 2 scans & 1 scan \\
\hline 1.35 & 1.87 & .2 .82 & 3.13 & 4.39 & 4.96 & 7.93 & 11.6 \\
\hline 1.59 & 1.64 & 2.51 & 3.9 & 5.53 & 5.14 & 7.24 & 11.6 \\
\hline 1.58 & 1.72 & 2.54 & 3.39 & 4.45 & 7.66 & 10 & 17.1 \\
\hline 1.54 & 1.8 & 2.83 & 3.23 & 5.51 & 6.81 & 9.5 & 15.3 \\
\hline \multirow[t]{7}{*}{1.33} & 1.85 & 2.45 & 3.42 & 5.13 & 8.61 & 10 & 14.2 \\
\hline & & & 3.87 & 4.92 & 7.23 & 7.62 & 12.2 \\
\hline & & & 3.73 & 5.75 & 6.33 & 9.72 & 13.5 \\
\hline & & & 3.56 & 3.54 & 6.52 & 9.63 & 14.5 \\
\hline & & & 3.54 & 5.49 & 6.38 & 9.18 & 13.4 \\
\hline & & & 3.54 & 4.5 & 7.47 & 10.9 & 12.2 \\
\hline & & & 3.88 & 4.13 & 7.43 & $\$ 1.1$ & 12.8 \\
\hline \multirow{2}{*}{\multicolumn{8}{|c|}{ Path Averaged Data (ppo). }} \\
\hline & & & & & & & \\
\hline 129 scans & 64 scans & 32 scans & 16 scans & 8 scans & 4 scans & $2 \operatorname{scans}$ & 1 scan \\
\hline 135 & 187 & 282 & 313 & 439 & 496 & 793 & 1160 \\
\hline 159 & 164 & 251 & 390 & 553 & 514 & 724 & 1160 \\
\hline 158 & 172 & 254 & 339 & 445 & 766 & 1000 & 1710 \\
\hline 154 & 180 & 283 & 323 & 551 & 681 & 950 & 1530 \\
\hline \multirow[t]{8}{*}{133} & 185 & 245 & 342 & 513 & 861 & 1000 & 1420 \\
\hline & & & 387 & 492 & 723 & 762 & 1220 \\
\hline & & & 373 & 575 & 633 & 972 & 1350 \\
\hline & & & 356 & 354 & 652 & 963 & 1450 \\
\hline & & & 354 & 549 & 638 & 918 & 1340 \\
\hline & & & 354 & 450 & 747 & 1090 & 1220 \\
\hline & & & 388 & 413 & 743 & 1110 & 1280 \\
\hline & & & & & & & \\
\hline Mean & Mean & Mean & Mean & Mean & Mean & Mean & Mean \\
\hline 148 & 178 & 263 & 356 & 485 & 678 & 935 & 1349 \\
\hline $195 \% 01$ & $195 \%$ C1) & $195 \%$ C & $195 \% \mathrm{Cll}$ & $195 \% \mathrm{Cl}$ & $195 \% \mathrm{Cl}$ & $195 \% \mathrm{Cli}$ & $(95 \% \mathrm{Cl}$ \\
\hline$(132-164)$ & $(166-190)$ & $(241-286)$ & $(339-374)$ & $(438.532)$ & $(605-750)$ & $(850-1020)$ & $(1235-1463)$ \\
\hline
\end{tabular}




\begin{tabular}{|c|c|c|c|c|c|c|c|}
\hline \multicolumn{8}{|c|}{ MOLs catculated by ETG sotiware: 3 -methyituran. $50 \mathrm{~m}$ patnlength. 3 sigma values } \\
\hline & & & & & & & \\
\hline \multicolumn{8}{|c|}{ Path Integrated Dala (pom-m\} } \\
\hline 128 scans & 64 scans & $32 \operatorname{scans}$ & 16 scans & 8 scans & 4 scans & $2 \operatorname{scans}$ & 1 scan \\
\hline 0.407 & 0.67 & 1.49 & 1.65 & 2.44 & 3.08 & 3.95 & 6.95 \\
\hline 0.529 & 0.673 & 1.19 & 1.62 & 2.31 & 2.49 & 4.17 & 7.24 \\
\hline 0.692 & 0.683 & 1.22 & 1.29 & 2.62 & 3.54 & 3.1 & 6.21 \\
\hline 0.559 & 0.797 & 1.01 & 1.23 & 2.48 & 2.49 & 3.72 & 4.27 \\
\hline \multirow[t]{2}{*}{0.6} & 0.599 & 1.12 & 1.48 & 1.86 & 3.56 & 3.69 & 6.4 \\
\hline & & & 1.36 & 2.07 & 2.72 & 4.72 & 6.44 \\
\hline \multirow[t]{6}{*}{-} & & & 1.48 & 1.81 & 3.53 & 4.53 & 5.74 \\
\hline & & & 1.81 & 1.72 & 3.75 & 4.38 & 4.96 \\
\hline & & & 1.37 & 2.11 & 3.24 & 5.65 & 7.26 \\
\hline & & & 1.48 & 1.53 & 2.76 & 3.57 & 7.11 \\
\hline & & & 1.53 & 2.09 & 3.14 & 4.05 & 7.41 \\
\hline & & & & & & & \\
\hline \multicolumn{8}{|c|}{ Path Averaged Data (ppb) } \\
\hline 128 scans & 64 scans & 32 scans & 16 sc3ns & 8 scans & 4 scans & 2 scans & 1 scan \\
\hline 8.14 & 13.4 & 29.8 & 33 & 48.8 & 61.6 & 79 & 139 \\
\hline 10.58 & 13.46 & 23.8 & 32.4 & 46.2 & 49.8 & 83.4 & 144.8 \\
\hline 13.84 & 13.66 & 24.4 & 25.8 & 52.4 & 70.8 & 62 & 124.2 \\
\hline 11.18 & 15.94 & 20.2 & 24.6 & 49.6 & 49.8 & 74.4 & 85.4 \\
\hline \multirow[t]{8}{*}{12} & 11.98 & 22.4 & 29.6 & 37.2 & 71.2 & 73.8 & 128 \\
\hline & & & 27.2 & 41.4 & 54.4 & 94.4 & 128.8 \\
\hline & & & 29.6 & 36.2 & 70.6 & 90.6 & 114.8 \\
\hline & & & 36.2 & 34.4 & 75 & 87.6 & 99.2 \\
\hline & & & 27.4 & 42.2 & 64.8 & 113 & 145.2 \\
\hline & & & 29.6 & 30.6 & 55.2 & 71.4 & 142.2 \\
\hline & & & 30.6 & 41.8 & 62.8 & 81 & 148.2 \\
\hline & & & & & & & \\
\hline Mean & Mean & Mean & Mean & Mean & Mean & Mean & Mean \\
\hline 11 & 14 & 24 & 30 & 42 & 62 & 83 & 127 \\
\hline $195 \%$ C.1) & $195 \%<11$ & $195 \% \mathrm{Cl}$ & $195 \% \mathrm{Ci}$ & $(95 \% \times 1)$ & $195 \%$ C 11 & $195 \% \mathrm{CN}$ & $195 \% \mathrm{CL}$ \\
\hline$(8-14)$ & $(12.15)$ & $(20.29)$ & $(27-32)$ & $(37-47)$ & $(56-58)$ & $(74-92)$ & $(114.141)$ \\
\hline
\end{tabular}




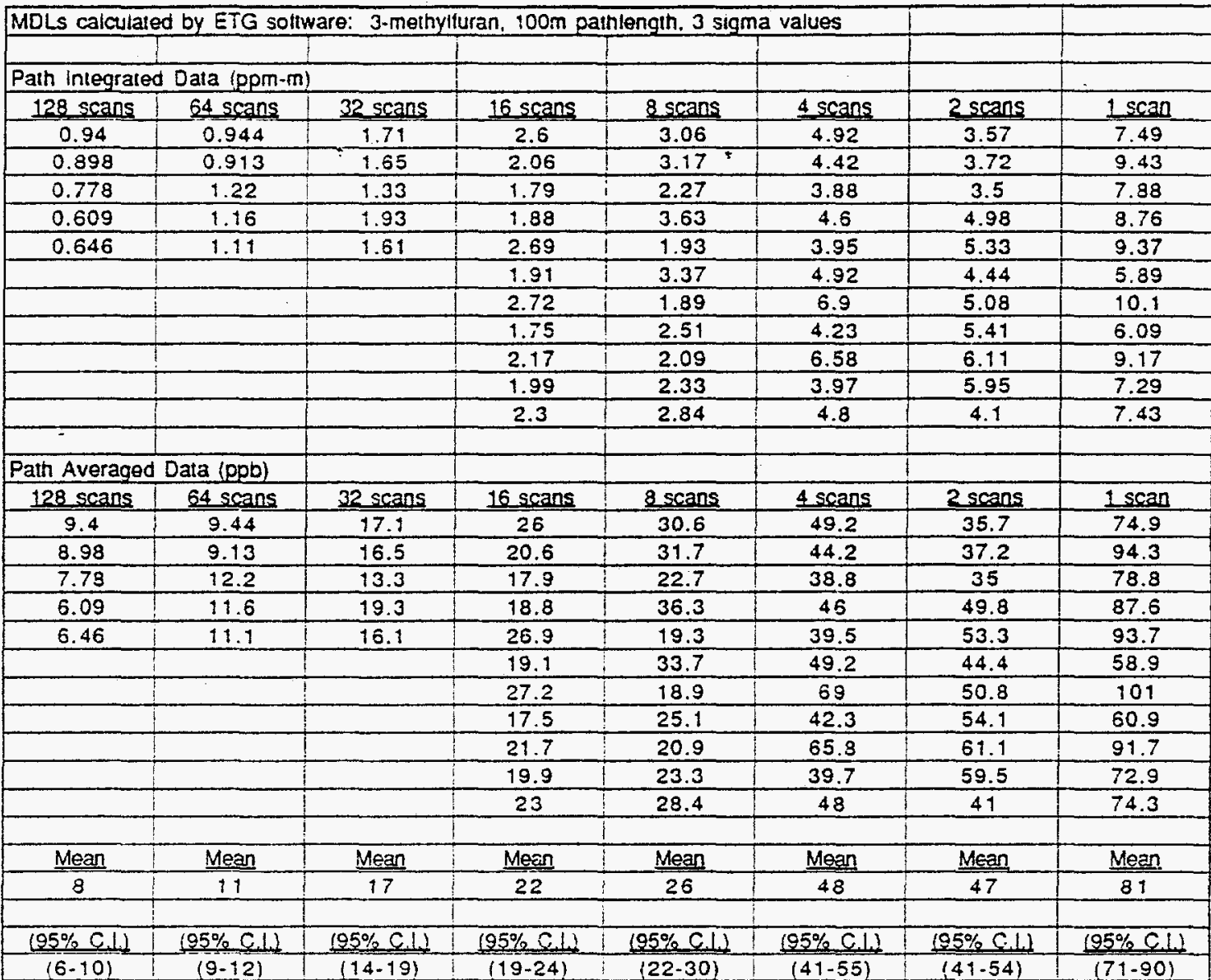




\begin{tabular}{|c|c|c|c|c|c|c|c|}
\hline \multicolumn{6}{|c|}{ MDLs calculaled by ETG solfware: $1-$ octen-3-01, $10 \mathrm{~m}$ pathlength, 3 sigma values } & & \\
\hline & & & & 1 & & & \\
\hline \multicolumn{8}{|c|}{ Path Integrated Data (ppm-m) } \\
\hline 128 scans & 24 scans & 32 scans & 16 scans & Bscans & 4 scans & 2 scans & 1 scan \\
\hline 5.52 & 3.4 & 5.33 & 5.82 & 9.32 & 12.4 & 15.8 & 14.8 \\
\hline 5.31 & 2.88 & 3.83 & 5.9 & 8.18 & 12.3 & 14.3 & 28.5 \\
\hline 4.02 & 4.36 & 5.5 & 9.16 & 9.06 & 13.8 & 21.4 & 22.1 \\
\hline 4.78 & 2.41 & 5.53 & 7.71 & 10.7 & 14.7 & 18.7 & 28.8 \\
\hline \multirow[t]{8}{*}{2.72} & 4.05 & 6.08 & 7.1 & 10.8 & 15.9 & 16.8 & 33.7 \\
\hline & & & 7.93 & 12 & 11.1 & 14.5 & \\
\hline & & & 9.17 & 11.8 & 16.9 & 18.7 & \\
\hline & & & 7.61 & 15.5 & 10.4 & 15 & \\
\hline & & & 9.52 & 11.6 & 14 & 17.1 & \\
\hline & & & 7.2 & 13.9 & 14.4 & 17.4 & \\
\hline & & & 6.45 & 12 & 14.7 & 20.8 & \\
\hline & & & & $!$ & & & \\
\hline \multicolumn{8}{|c|}{ Path Averaged Data (ppo) } \\
\hline 128 scans & 64 scans & 32 scans & 16 scans & 8 scans & 4 scans & 2 scans & 1 scan \\
\hline 552 & 340 & 533 & 582 & 932 & 1240 & 1580 & 1480 \\
\hline 531 & 288 & 383 & 590 & 818 & 1230 & 1430 & 2850 \\
\hline 402 & 436 & 550 & 915 & 906 & 1380 & 2140 & 2210 \\
\hline 478 & 241 & 553 & 771 & 1070 & 1470 & 1870 & 2880 \\
\hline \multirow[t]{8}{*}{272} & 405 & 608 & 710 & 1080 & 1590 & 1680 & 3370 \\
\hline & & & 793 & 1200 & 1110 & 1450 & \\
\hline & & & 917 & 1180 & 1690 & 1870 & \\
\hline & & & 761 & 1550 & 1040 & 1500 & \\
\hline & & & 952 & 1160 & 1400 & 1710 & \\
\hline & & & 720 & 1390 & 1440 & 1740 & \\
\hline & & & 645 & 1200 & 1470 & 2080 & \\
\hline & & & & $!$ & & & \\
\hline Mean & Mean & Mean & Mean & Mean & Mean & Mean & Mean \\
\hline 447 & 342 & 525 & 760 & 1135 & 1369 & 1732 & 2558 \\
\hline & & & & & & & \\
\hline$(95 \%, 1)$ & $195 \%<11$ & $95 \% \mathrm{CL}$ & $(95 \% \mathrm{c}, 1)$ & $195 \% \mathrm{cll}$ & $(95 \% \mathrm{C} 1)$ & $195 \%<11$ & $(95 \% \mathrm{c})$ \\
\hline$(306-588)$ & $(242.442)$ & $(421-630)$ & $(673-846)$ & $(993-1278)$ & $(1236-1502)$ & $(1571-1893)$ & $(2068-3048)$ \\
\hline
\end{tabular}




\begin{tabular}{|c|c|c|c|c|c|c|c|}
\hline MDLs calculal & 1 by ETG so & are: 1-octen & $50 \mathrm{~m} \mathrm{pa}$ & th. 3 sigm & IUes & & \\
\hline & & & & & & & \\
\hline Path integrate & Data $(\mathrm{ppm}-1$ & & & & & & \\
\hline 128 scans & 64 scans & 32 scans & 16 scans & 8 scans & $₫$ scans & 2 scans & 1 scan \\
\hline 1.99 & 2.12 & 3.26 & 3.17 & 6.42 & 7.6 & 9.39 & 15.6 \\
\hline 1.72 & 2.03 & 3.31 & 2.95 & 6.23 & 8.26 & 9.12 & 16.9 \\
\hline 1.67 & 1.83 & 3.45 & 4.38 & 6.99 & 9.58 & 12.5 & 16.5 \\
\hline 1.83 & 2.13 & 2.71 & 3.55 & 6.61 & 8.15 & 13.2 & 16.6 \\
\hline 1.29 & 2.46 & 3.13 & 3.27 & 6.19 & 8.24 & 13.6 & 15.8 \\
\hline & & & 3.74 & 7.45 & 9.67 & 15.3 & 12.7 \\
\hline & & & 4.02 & 8.19 & 8.69 & 11.6 & 12.9 \\
\hline & & & 3.29 & 7.07 & 7.2 & 9.74 & 16 \\
\hline & & & 3.46 & 6.38 & 8.51 & 12.1 & 12.2 \\
\hline & & & 4.09 & 6.64 & 9.96 & 9.07 & 12.5 \\
\hline & & & 4.29 & 3.88 & 8 & 10.5 & 14.5 \\
\hline Path Average & Data (ppo) & & & & & & \\
\hline 128 scans & 64 scans & 32 scans & 16 scans & 8 scans & 4 scans & 2 scans & 1 scan \\
\hline 39.8 & 42.4 & 65.2 & 63.4 & 128.4 & 152 & 187.8 & 312 \\
\hline 34.4 & 40.6 & 66.2 & 59 & 124.6 & 165.2 & 182.4 & 338 \\
\hline 33.4 & 36.6 & 69 & 87.6 & 139.8 & 191.6 & 250 & 330 \\
\hline 36.6 & 42.6 & 54.2 & 71 & 132.2 & 163 & 264 & 332 \\
\hline 25.3 & 49.2 & 62.6 & 65.4 & 123.8 & 164.8 & 272 & 316 \\
\hline & & & 74.8 & 149 & 193.4 & 306 & 254 \\
\hline & & & 80.4 & 163.8 & 173.8 & 232 & 258 \\
\hline & & & 65.8 & 141.4 & 144 & 194.8 & 320 \\
\hline & & & 69.2 & 127.6 & 170.2 & 242 & 244 \\
\hline & & & 81.8 & 132.8 & 199.2 & 181.4 & 250 \\
\hline & & & 85.8 & 77.6 & 160 & 210 & 290 \\
\hline & & & & & & & \\
\hline Mean & Mean & Mean & Mean & Mean & Mean & Mean & Mean \\
\hline 34 & 42 & 63 & 73 & 131 & 171 & 229 & 295 \\
\hline $195 \% \mathrm{CL}$ & $195 \%$ C.L & $95 \% \mathrm{CL}$ & $195 \% 011$ & $195 \% \mathrm{CW}$ & $195 \% \mathrm{Ch}$ & $195 \% \mathrm{Cl}$ & $95 \% \mathrm{CL}$ \\
\hline$(28.40)$ & $(37.48)$ & $(56-70)$ & $(67-80)$ & $(117.145)$ & $(159-182)$ & $(201-257)$ & $(270-320)$ \\
\hline
\end{tabular}




\begin{tabular}{|c|c|c|c|c|c|c|c|}
\hline \multicolumn{8}{|c|}{ MOLS calculated by ETG soltware: $1-0 c t e n-3-01,100 \mathrm{~m}$ painlength, 3 sigma values } \\
\hline & & & & & & & \\
\hline \multicolumn{8}{|c|}{ Path Integrated Data (ppm-m) } \\
\hline 128 scans & 64 scans & 32 scans & $16 \operatorname{scans}$ & 8 scans & 4 scans & 2 scans & 1 scan \\
\hline 2.19 & 2.52 & 3.88 & 3.81 & 5.98 & 6.53 & .9 .21 & 12.1 \\
\hline 2.28 & 2.28 & 4.52 & 2.75 & 6.45 & 5.23 & 9.64 & 13.6 \\
\hline 2.25 & 1.71 & 5.17 & 4.49 & 4.09 & 7.09 & 9.13 & 11.9 \\
\hline 2.2 & 2.23 & 5.92 & 3.45 & 5.47 & 5.86 & 9.69 & 14.3 \\
\hline \multirow[t]{8}{*}{2.31} & 2.15 & 3.95 & 4.26 & 4.83 & 6.96 & 10 & 15.3 \\
\hline & & & 4.4 & 5.27 & 7.25 & 8.86 & 8.51 \\
\hline & & & 3.19 & 4.97 & 6.21 & 9.67 & 14.3 \\
\hline & & & 3.73 & 5.55 & 7.06 & 10 & 12.7 \\
\hline & & & 4.68 & 6.04 & 5.59 & 10.4 & 15.1 \\
\hline & & & 2.96 & 5.21 & 7.06 & 12.1 & 14.7 \\
\hline & & & 4.48 & 5.01 & 6.4 & 11.9 & 17.3 \\
\hline & & & & & & & \\
\hline \multicolumn{8}{|c|}{ Path Averaged Dala (ppb) } \\
\hline 128 scans & 64 scans & 32 scans & 16 scans & 8 scans & 4 scans & $2 \operatorname{scans}$ & 1 scan \\
\hline 21.9 & 25.2 & 38.8 & 38.1 & 59.8 & 65.3 & 92.1 & 121 \\
\hline 22.8 & 22.8 & 45.2 & 27.5 & 64.5 & 52.3 & 96.4 & 136 \\
\hline 22.5 & 17.1 & 51.7 & 44.9 & 40.9 & 70.9 & 91.3 & 119 \\
\hline 22 & 22.3 & 59.2 & 34.5 & 54.7 & 58.6 & 96.9 & 143 \\
\hline \multirow[t]{7}{*}{23.1} & 21.5 & 39.5 & 42.6 & 48.3 & 69.6 & 100 & 153 \\
\hline & & & 44 & 52.7 & 72.5 & 88.6 & 85.1 \\
\hline & & & 31.9 & 49.7 & 62.1 & 96.7 & 143 \\
\hline & & & 37.3 & 55.5 & 70.6 & 100 & 127 \\
\hline & & & 46.8 & 60.4 & 55.9 & 104 & 151 \\
\hline & & & 29.6 & 52.1 & 70.6 & 121 & 147 \\
\hline & & & 44.8 & 50.9 & 64 & 119 & 173 \\
\hline & & & & & & & \\
\hline Mean & Mean & Mean & Mean & Mean & Mean & Mean & Mean \\
\hline 23 & 22 & 47 & 38 & 54 & 65 & 101 & 136 \\
\hline $195 \%(1)$ & $195 \% \mathrm{Cl}$ & $195 \% \times 11$ & $195 \% \mathrm{CH}$ & $195 \% 011$ & $195 \% \mathrm{Cl}$ & $195 \% \mathrm{Cl} 11$ & $95 \%$ C.11 \\
\hline$(22-23)$ & $(18-26)$ & $(36-58)$ & $(34-43)$ & $(49-58)$ & $(60.69)$ & $(93.108)$ & $(121-152)$ \\
\hline
\end{tabular}




\begin{tabular}{|c|c|c|c|c|}
\hline \multicolumn{5}{|c|}{ MDLs: Humidity adjusted from the original $62 \%$ R.H. @ $22^{\circ} \mathrm{C}$} \\
\hline & & & & \\
\hline \multicolumn{5}{|c|}{ Relative Humidity adjusted to $34 \% @ 22^{\circ} \mathrm{C}(10 \mathrm{~m})$} \\
\hline & & & & \\
\hline 3-methylfuran & & & $1-$ octen-3-ol & \\
\hline & & & & \\
\hline$(p p m-m)$ & (ppb) & & $(p p m-m)$ & $(p p b)$ \\
\hline 128 scans & 128 scans & & 128 scans & 128 scans \\
\hline 1.21 & 121 & & 5.09 & 509 \\
\hline 1.44 & 144 & & 5.55 & 555 \\
\hline 1.35 & 135 & & 4.14 & 414 \\
\hline 1.4 & 140 & & 4.67 & 467 \\
\hline \multirow[t]{7}{*}{1.22} & 122 & & 2.48 & 248 \\
\hline & & & & \\
\hline & Mean & & & Mean \\
\hline & 132 & & & 439 \\
\hline & $195 \%$ C. 1.1 & & & $(95 \%$ C. 1.1$)$ \\
\hline & $(119-145)$ & & & $(291-586)$ \\
\hline & & & & \\
\hline \multicolumn{5}{|c|}{ Relative Humidity adjusted to $77 \%$ @ $22^{\circ} \mathrm{C}(10 \mathrm{~m})$} \\
\hline & & & & \\
\hline \multirow{2}{*}{ 3-methylfuran } & & & 1-ocien-3-01 & \\
\hline & & & & \\
\hline (ppm-m) & $(\mathrm{ppb})$ & & $(\mathrm{ppm}-\mathrm{m})$ & (ppb) \\
\hline 128 scans & 128 scans & & 128 scans & 128 scans \\
\hline 3.31 & 331 & & 5.5 & 550 \\
\hline 2.27 & 227 & & 5.53 & 553 \\
\hline 4.44 & 444 & & 4.23 & 423 \\
\hline 4.44 & 444 & & 4.64 & 464 \\
\hline \multirow[t]{6}{*}{2.49} & 249 & & 2.55 & 255 \\
\hline & & & & \\
\hline & Mean & & & Mean \\
\hline & 339 & & & 449 \\
\hline & $(95 \%$ C. 1.1 & & & $(95 \%$ C.1. \\
\hline & $(211-467)$ & & & $(298-600)$ \\
\hline
\end{tabular}




\begin{tabular}{|c|c|c|c|c|}
\hline \multicolumn{5}{|c|}{ MDLs: Humidity adjusted from the original $62 \%$ R.H. @ $22^{\circ} \mathrm{C}$} \\
\hline & & & & \\
\hline \multicolumn{5}{|c|}{ Relative Humidity adjusted to $34 \% @ 22^{\circ} \mathrm{C}(50 \mathrm{~m})$} \\
\hline & & & & - \\
\hline 3-methylfuran & & & 1-octen-3-ol & \\
\hline & & & & \\
\hline$(p p m-m)$ & (ppb) & & $(p p m-m)$ & (ppb) \\
\hline 128 scans & 128 scans & & 128 scans & 128 scans \\
\hline 0.364 & 7.28 & & 2 & 40 \\
\hline 0.559 & 11.18 & & 1.74 & 34.8 \\
\hline 0.928 & 18.56 & & 1.69 & 33.8 \\
\hline 0.766 & 15.32 & & 1.83 & 36.6 \\
\hline \multirow[t]{8}{*}{0.54} & 10.8 & & 1.29 & 25.8 \\
\hline & & & & \\
\hline & Mean & & & Mean \\
\hline & 13 & & & 34 \\
\hline & $95 \%$ C.1.) & & & $(95 \%$ C.1. \\
\hline & $(7-18)$ & & & $(28-41)$ \\
\hline & & & & \\
\hline & & & & \\
\hline \multicolumn{5}{|c|}{ Relative Humidity adjusted to $77 \% @ 22^{\circ} \mathrm{C}(50 \mathrm{~m})$} \\
\hline & & & & \\
\hline \multirow[t]{2}{*}{ 3-methylfuran } & & & 1-octen-3-ol & \\
\hline & & & & \\
\hline$(p p m-m)$ & (ppb) & & $(\mathrm{ppm}-\mathrm{m})$ & $(p p b)$ \\
\hline 128 scans & 128 scans & & 128 scans & 128 scans \\
\hline 0.693 & 13.86 & & 1.98 & 39.6 \\
\hline 1.14 & 22.8 & & 1.71 & 34.2 \\
\hline 1.64 & 32.8 & & 1.65 & 33 \\
\hline 0.874 & 17.48 & & 1.83 & 36.6 \\
\hline \multirow[t]{6}{*}{1.46} & 29.2 & & 1.28 & 25.6 \\
\hline & & & & \\
\hline & Mean & & & Mean \\
\hline & 23 & & & 34 \\
\hline & $(95 \%$ C.I. & & & $195 \%$ C.I. \\
\hline & $(13-33)$ & & & $(27-40)$ \\
\hline
\end{tabular}




\begin{tabular}{|c|c|c|c|c|}
\hline \multirow{2}{*}{\multicolumn{5}{|c|}{ Relative Humidity adjusted to $34 \% @ 22^{\circ} \mathrm{C}(100 \mathrm{~m})$}} \\
\hline & & & & \\
\hline & & & - & \\
\hline 3-methvifuran & & & 1-octen-3-01 & \\
\hline & & & & \\
\hline$(\mathrm{ppm}-\mathrm{m})$ & (ppb) & & $(\mathrm{ppm}-\mathrm{m})$ & $(p p b)$ \\
\hline 128 scans & 128 scans & & 128 scans & 128 scans \\
\hline 0.806 & 8.06 & & 2.19 & 21.9 \\
\hline 0.815 & 8.15 & & 2.28 & 22.8 \\
\hline 0.907 & 9.07 & & 2.24 & 22.4 \\
\hline 0.507 & 5.07 & 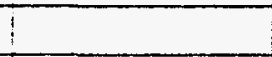 & 2.2 & 22 \\
\hline \multirow{8}{*}{0.688} & 6.88 & & 2.31 & 23.1 \\
\hline & & & & \\
\hline & Mean & & & Mean \\
\hline & 7 & & & 22 \\
\hline & $195 \%$ C.1.) & & & $(95 \%$ C.1.) \\
\hline & $(6-9)$ & & & $(22-23)$ \\
\hline & & & & \\
\hline & & & & \\
\hline \multicolumn{5}{|c|}{ Relative Humidity adjusted to $77 \%$ @ $22^{\circ} \mathrm{C}(100 \mathrm{~m})$} \\
\hline \multicolumn{5}{|c|}{\begin{tabular}{r|l|l|}
1 & & \\
\end{tabular}} \\
\hline \multirow[t]{2}{*}{ 3-methvlfuran } & & & 1-octen-3-0l & \\
\hline & & & & \\
\hline$(\mathrm{ppm}-\mathrm{m})$ & $(p p b)$ & & $(p p m-m)$ & $(p, p b)$ \\
\hline 128 scans & 128 scans & & 128 scans & 128 scans \\
\hline 4.78 & 47.8 & & 2.19 & 21.9 \\
\hline 11.6 & 116 & & 2.28 & 22.8 \\
\hline 10.4 & 104 & & 2.25 & 22.5 \\
\hline 61.7 & 617 & & 2.19 & 21.9 \\
\hline \multirow[t]{6}{*}{50.3} & 503 & & 2.3 & 23 \\
\hline & & & & \\
\hline & Mean & & & Mean \\
\hline & 278 & & & 22 \\
\hline & $195 \%$ C.1.) & & & $95 \%$ C.1. \\
\hline & $((-48)-603)$ & & & $(22-23)$ \\
\hline
\end{tabular}


Appendix B

Example Spectra

Single Beam Spectrum

Absorbance Spectrum

Baseline Noise

Zero-Path Spectrum (single beam)

Water Vapor spectrum (absorbance) 


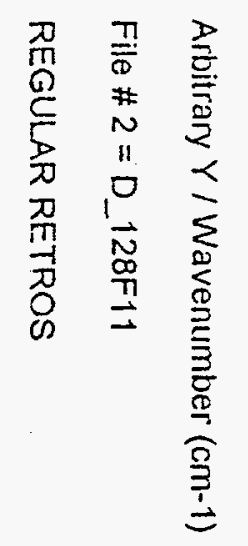

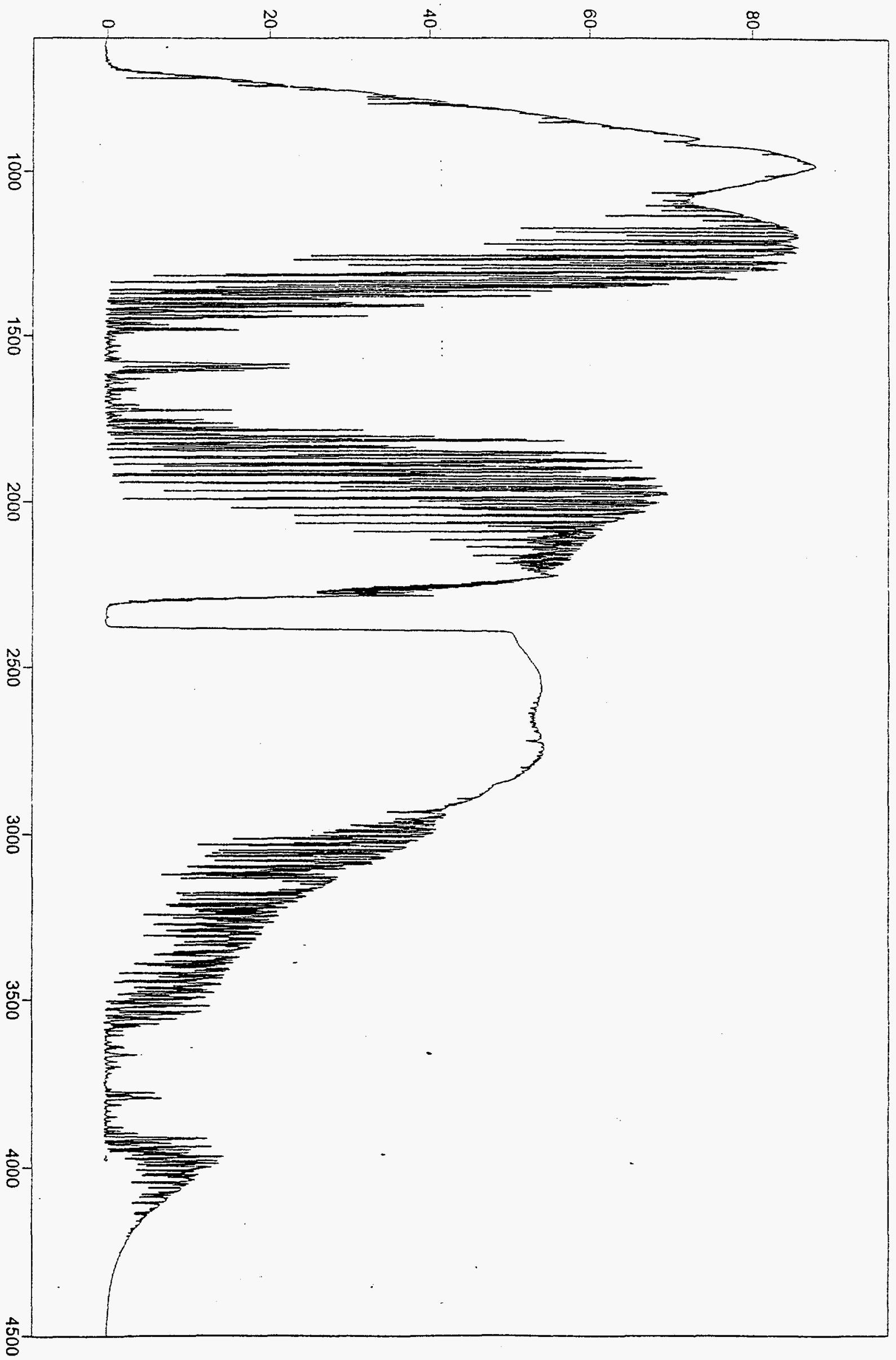



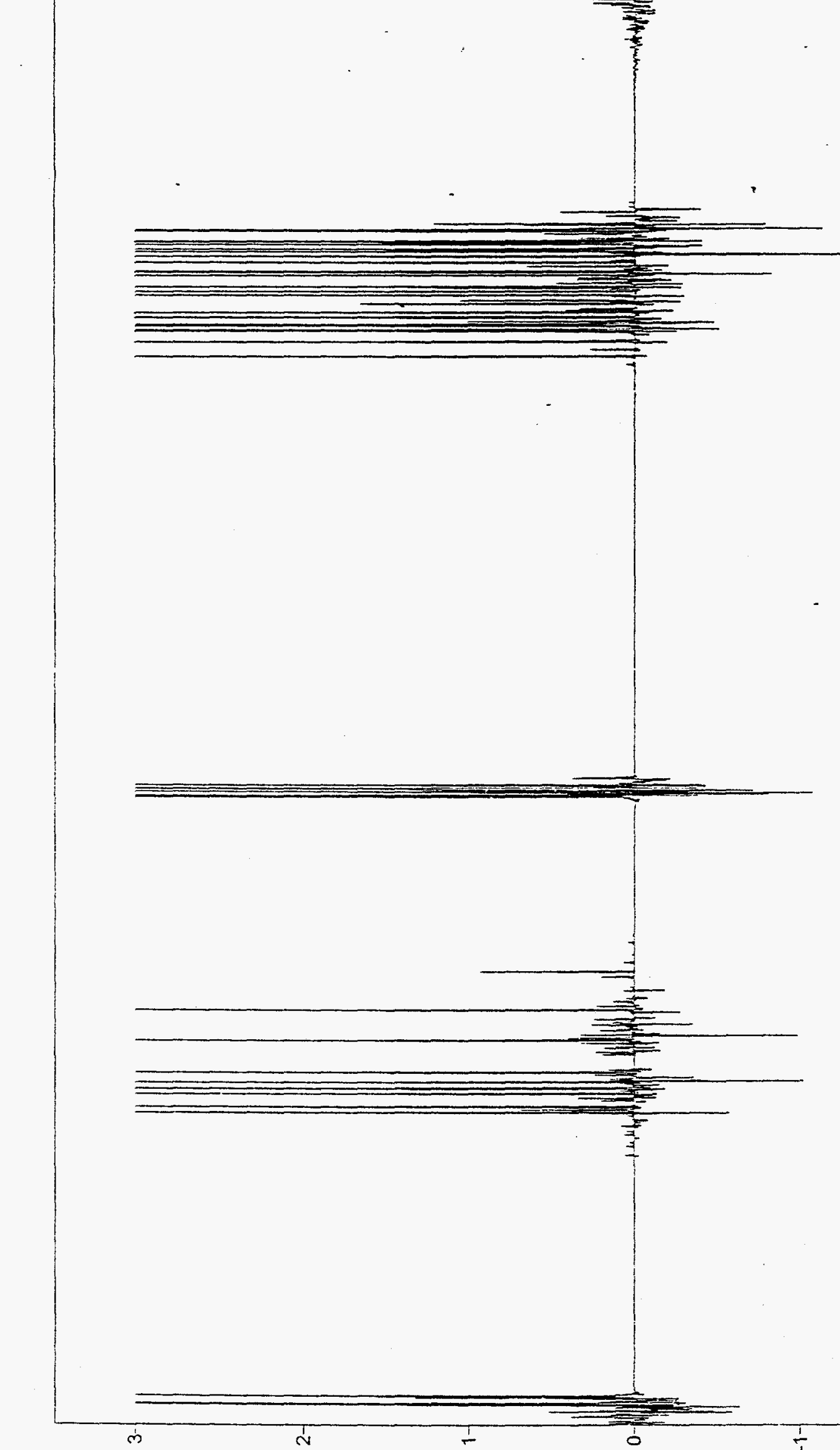

-8

-8

足

-8

8
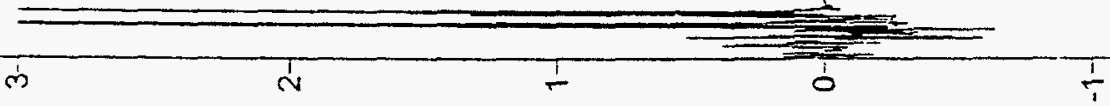

$\gamma$

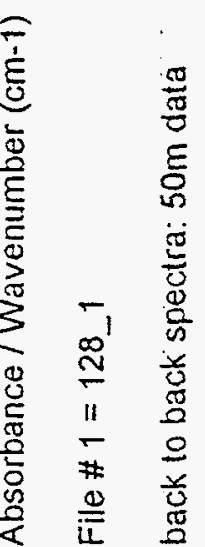




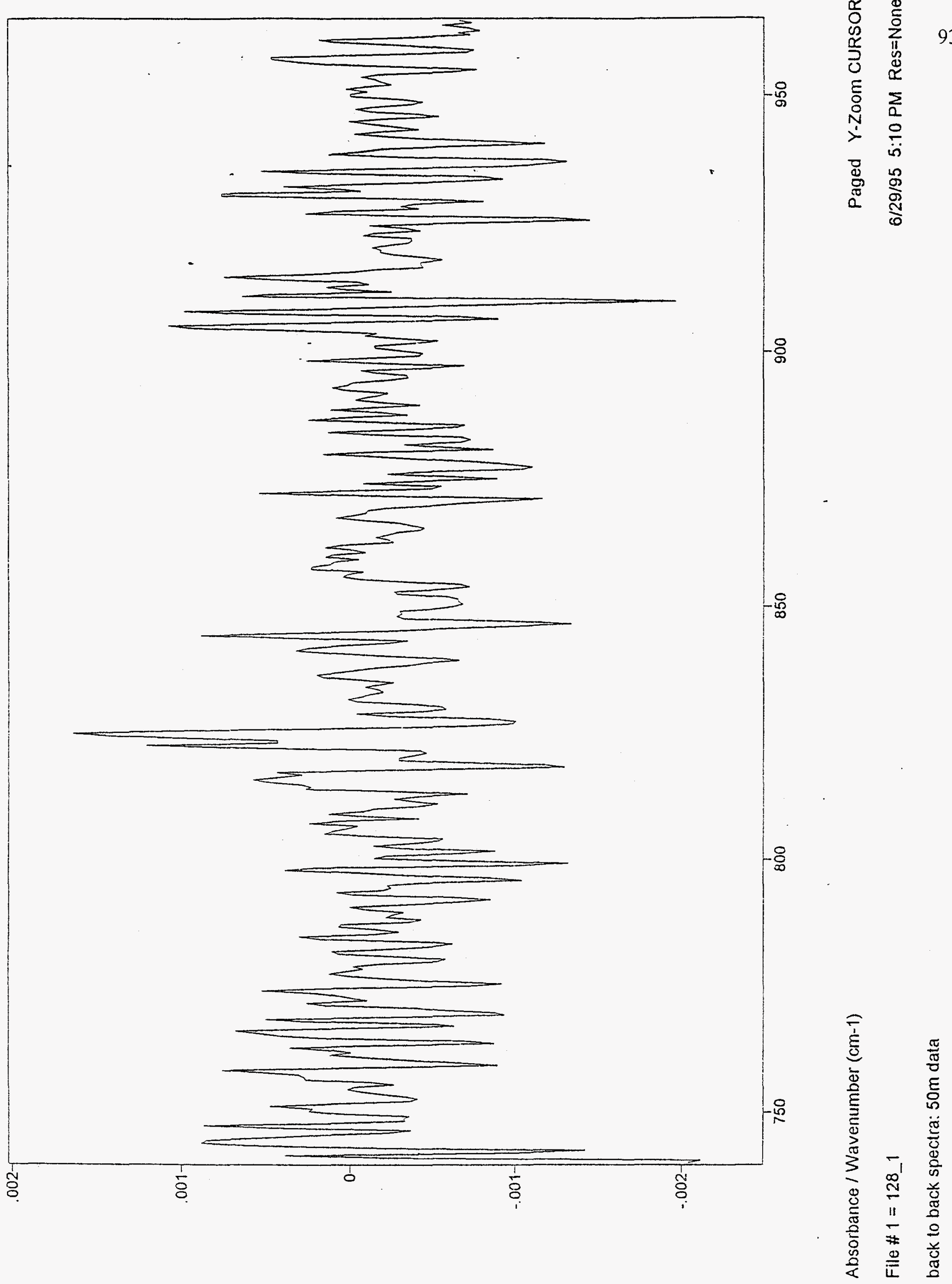




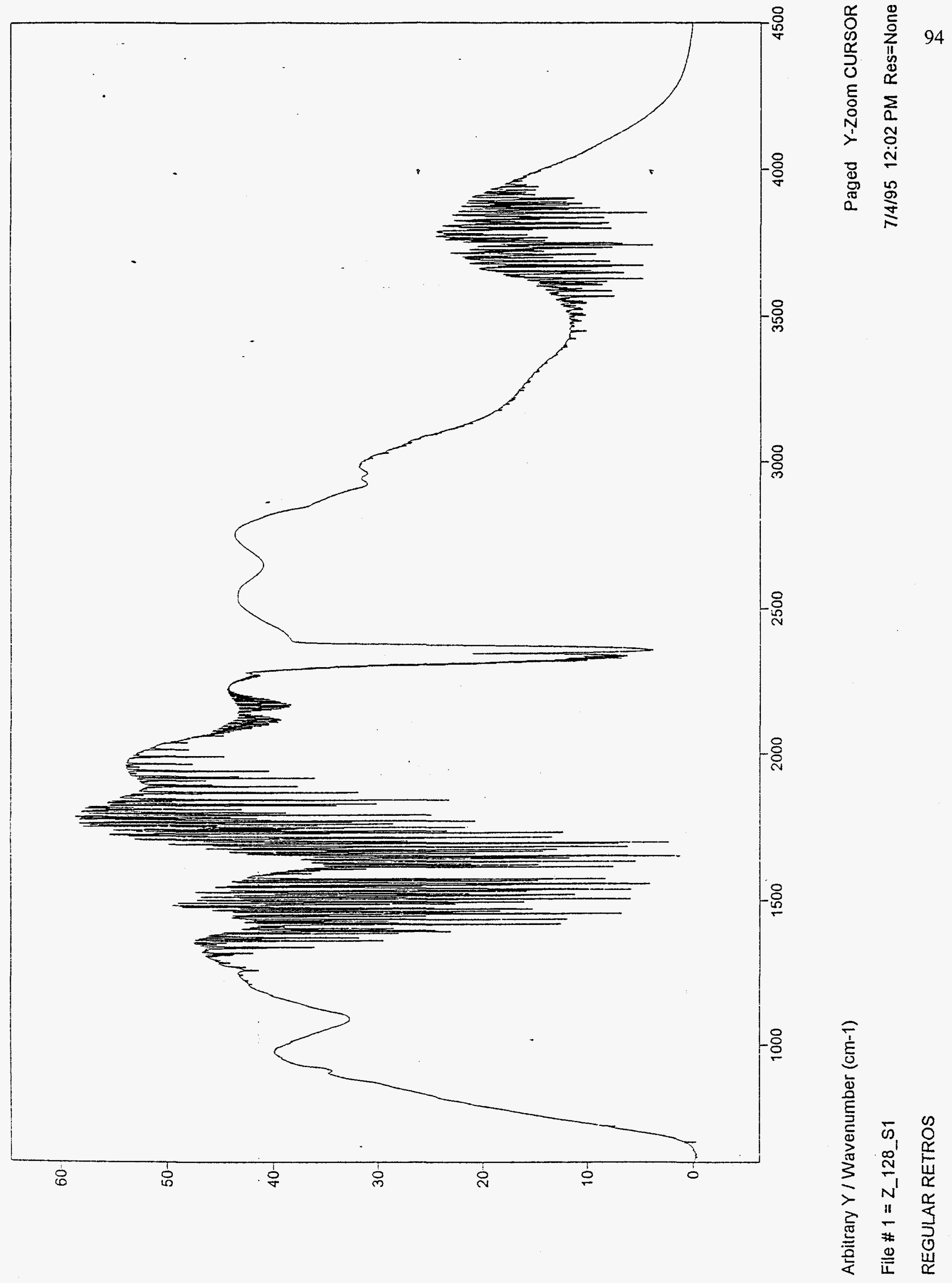




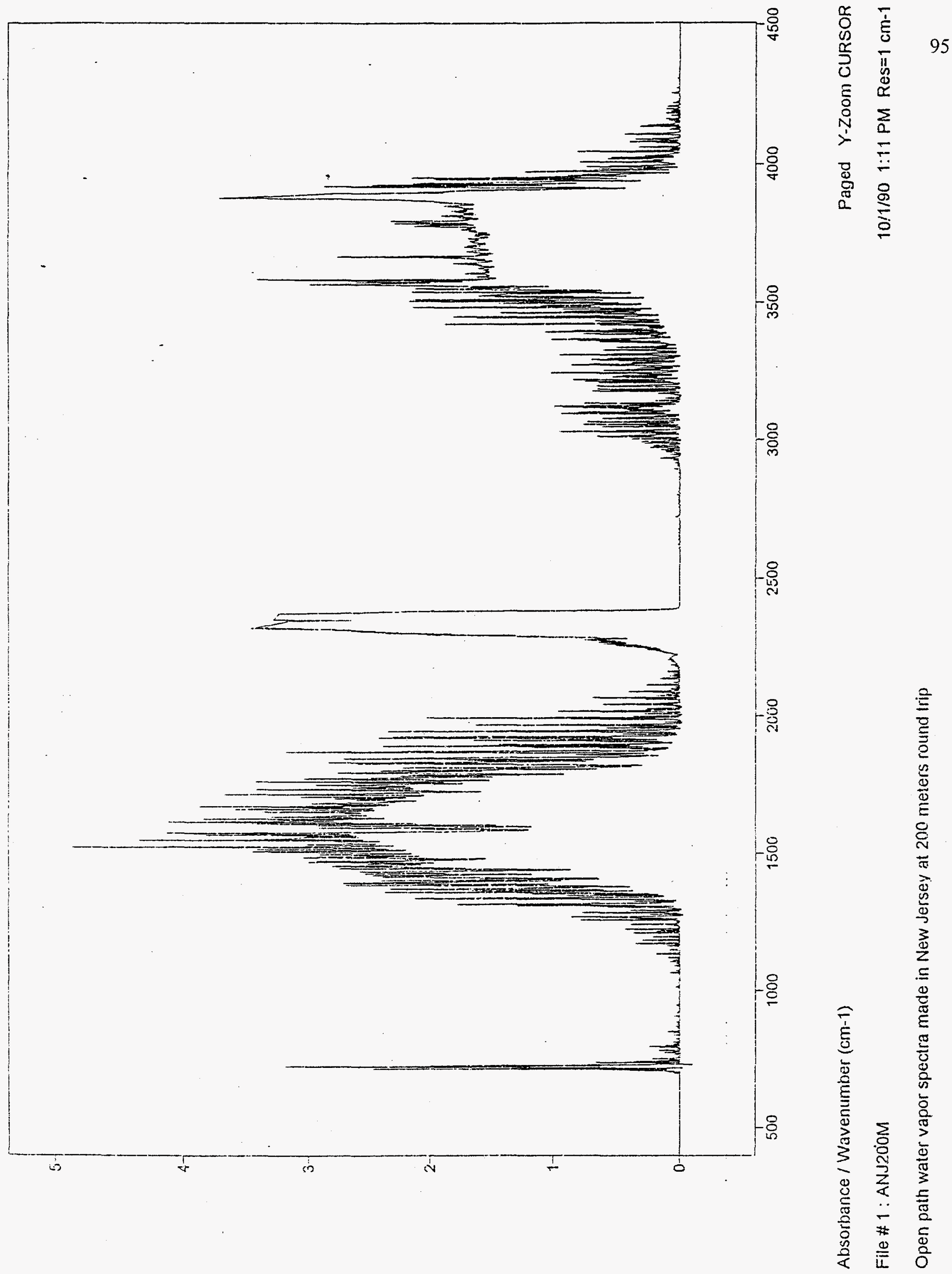

